\title{
Implementing the Panel Event Study
}

\author{
Autores: \\ Damian Clarke \\ Kathya Tapia Schythe
}




\title{
Implementing the Panel Event Study
}

\author{
Damian Clarke \\ Department of Economics \\ University of Chile \\ dclarke@fen.uchile.cl
}

\author{
Kathya Tapia Schythe \\ Department of Economics \\ University of Santiago de Chile \\ kathya.tapia@usach.cl
}

\begin{abstract}
Many studies estimate the impact of exposure to some quasi-experimental policy or event using a panel event study design. These models, as a generalized extension of 'difference-in-differences' or two-way fixed effect models, allow for dynamic lags and leads to the event of interest to be estimated, while also controlling for fixed factors (often) by area and time. In this paper we discuss the set-up of the panel event study design in a range of situations, and lay out a number of practical considerations for its estimation. We describe a Stata command eventdd that allows for simple estimation, inference, and visualization of event study models in a range of circumstances. We then provide a number of examples to illustrate eventdd's use and flexibility, as well as its interaction with various native Stata routines, and other relevant user-written libraries such as reghdfe and boottest.
\end{abstract}

Keywords: , event studies, difference-in-differences, estimation, inference, visualization

\section{Introduction}

Recent developments in quasi-experimental methods have brought increasing attention to panel event study models. Using data covering a panel of observations (such as states) over time, the design seeks to estimate the impact of some event which occurs, or 'switches on' in certain units and certain time periods. ${ }^{1}$ They seek to use as counterfactuals the areas in which the policy or event does not occur, or has not yet occurred. By considering the variation in outcomes around the adoption of the event compared with a baseline reference period, both event lags and leads are estimated, allowing for a clear visual representation of the event's causal impact provided that key identifying assumptions are met.

These methods have been borne out of older difference-in-differences, or two-way fixed effect models. These models often seek to examine the impact of natural experiments, where events are assigned to certain units due to some process beyond the control of the analyst but owing to environmental or political factors (among others), and as such, generally do not assume that assignment is random. Indeed, as we lay

\footnotetext{
${ }^{1}$ Throughout this paper, for expositional purposes we will refer to a geographical and a temporal dimension to these panel event study designs. However, we note that such models are suitable to other settings where one wishes to estimate the impact of an event which is assigned to certain units, and where there is some temporal variation in outcomes to be studied. For example, rather than being based on different geographical units at different times, these models could be based on an event occurring in different age groups at different times. All results discussed in this paper extend to settings such as these.
} 
out at more length in the following section, the key assumption underlying consistent estimation in event study models is that the occurrence of the event in a particular area is not systematically related to the changes in levels that will occur in the future.

These models are widely used in empirical analyses in a range of contexts, having been applied to (among many others themes) automotive plant closures and opioid overdoses (Venkataramani et al. 2020), family planning access and childhood economic circumstance (Bailey et al. 2018), health care reform and ambulatory care usage (Dimitrovová et al. 2020) and university reform and intergenerational mobility (Suhonen and Karhunen 2019). These cases suggest usage across a range of fields including social sciences, medicine and public health, and additional reviews of their frequency of use in a number of economic journals are provided in Abraham and Sun (2018); Roth (2019). A burgeoning literature has laid out a number of identification requirements in this setting (Freyaldenhoven et al. 2019; Borusyak and Jaravel 2018; Abraham and Sun 2018; Athey and Imbens 2018; Schmidheiny and Siegloch 2019). These methods can be used, with some restrictions, both in cases where events occur at the same time period in each unit, and in cases where the adoption of events is staggered. Indeed, Athey and Imbens (2018) refer to these as "Staggered Adoption Designs", although here we follow the more common nomenclature of panel event studies. ${ }^{2}$ Additionally, these methods are related to a much broader literature on staggered adoption of policies and the estimation of a single-coefficient model (de Chaisemartin and D'Haultfoeuille 2019; Callaway and Sant'Anna 2018; Goodman-Bacon 2018). While we briefly discuss these models in the methods section, our principal interest is on full panel-event study specifications which come with their own considerations.

In this paper we provide a discussion of these panel-event study models, and practical issues related to their estimation and to inference in these settings. We also present the eventdd command, a Stata command allowing for estimation and inference in event studies, as well as the graphical presentation of their results. ${ }^{3}$ This command can flexibly interact with both in-built Stata commands such as regress and xtreg, as well as the user-written regression command reghdfe which is highly convenient in two-way fixed effect models such as those described in this paper (Correia 2016). We provide discussion of both estimation and inference in event study models. As well as standard inference procedures such as robust and cluster-robust inference, the eventdd command allows for wild-bootstrap based inference respecting the clustered nature of the occurrence of events, and specifically the user-written boottest command (Roodman et al. 2019). After reviewing the theory behind panel event study models in section 2, we discuss the command syntax in section 3 , before documenting the command's usage, applied to a particular empirical example, in Section 4.

\footnotetext{
${ }^{2}$ An older literature, often related to the analyses of financial events and information shocks, refers to event studies. These event studies in finance are generally based on time-series observations, and have quite different properties to the panel event studies used in policy analysis that we discuss in this paper. A useful discussion of these finance-style event studies, and their application in Stata, is provided in Pacicco et al. (2018).

${ }^{3}$ An earlier version of this command is available as Clarke and Tapia Schythe (2020).
} 


\section{Methods}

\subsection{Estimation}

Consider a panel covering states $s$ and time periods $t$. We are interested in estimating the impact of the passage of an event which may occur at different times in different states. We will denote as Event $s$ a variable recording the time period $t$ in which the event is adopted in state $s$. Denoting the outcome of interest as $y_{s t}$, the panel event study specification can be written $\mathrm{as}^{4}$ :

$$
y_{s t}=\alpha+\sum_{j=2}^{J} \beta_{j}(\operatorname{Lag} j)_{s t}+\sum_{k=1}^{K} \gamma_{k}(\operatorname{Lead} k)_{s t}+\mu_{s}+\lambda_{t}+X_{s t}^{\prime} \Gamma+\varepsilon_{s t} .
$$

Here $\mu_{s}$ and $\lambda_{t}$ are state and time fixed effects, $X_{s t}$ are (optionally) time-varying controls, and $\varepsilon_{s t}$ is an unobserved error term. In equation 1, lags and leads to the event of interest are defined as follows:

$$
\begin{aligned}
(\operatorname{Lag} J)_{s t} & =\mathbb{1}\left[t \leq \text { Event }_{s}-J\right] \\
(\operatorname{Lag} j)_{s t} & =\mathbb{1}\left[t=\text { Event }_{s}-j\right] \text { for } j \in\{1, \ldots, J-1\}, \\
(\text { Lead } k)_{s t} & =\mathbb{1}\left[t=\text { Event }_{s}+k\right] \text { for } k \in\{1, \ldots, K-1\}, \\
(\operatorname{Lead} K)_{s t} & =\mathbb{1}\left[t \geq \text { Event }_{s}+K\right] .
\end{aligned}
$$

Lags and leads are thus binary variables indicating that the given state was a given number of periods away from the event of interest in the respective time period. $J$ and $K$ lags and leads are included respectively, and, as indicated in equations 2 and 5 , final lags and leads "accumulate" lags or leads beyond $J$ and $K$ periods. A single lag or lead variable is omitted to capture the baseline difference between areas where the event does and does not occur. In specification 1, as standard, this baseline omitted case is the first lag, where $j=1$.

\footnotetext{
${ }^{4}$ There are a number of ways to specify such a model. Slightly different notations are used by Schmidheiny and Siegloch (2019) who define the model as:

$$
y_{s t}=\sum_{j=\underline{j}}^{\bar{j}} \beta_{j} b_{s t}^{j}+\mu_{s}+\lambda_{t}+\varepsilon_{s t}
$$

where

$$
b_{s t}^{j}= \begin{cases}\mathbb{1}\left[t \leq \text { Event }_{s}+j\right] & \text { if } j=\bar{j} \\ \mathbb{1}\left[t=\text { Event }_{s}+j\right] & \text { if } \bar{j}<\bar{j}<\bar{j} \\ \mathbb{1}\left[t \geq \text { Event }_{s}+j\right] & \text { if } \bar{j}=\bar{j},\end{cases}
$$

and where $j$ is equivalent to our definition of $J$ and $\bar{j}$ is equivalent to our $L$. In the case of Freyaldenhoven et al. $(201 \overline{9})$, they define a version of this model as:

$$
y_{s t}=\delta_{-K+}\left(1-z_{s, t+(K-1)}\right)+\delta_{L+} z_{s, t-L}+\sum_{k=-(L-1)}^{K-1} \delta_{-k} \Delta z_{s, t+k}+\mu_{s}+\lambda_{t}+\varepsilon_{s t},
$$

where $z_{s t} \equiv$ PostEvent $t_{s t}$ as defined in Table $1, z_{s, t+k}$ and $z_{s, t-k}$ refer to lags and leads of this variable respectively, and $\Delta$ refer to the first difference of these lag/lead terms. These models, and that laid out in equations $1-5$ are equivalent.
} 


\begin{tabular}{|c|c|c|c|c|c|c|c|c|c|c|c|}
\hline $\begin{array}{l}\text { State } \\
(\mathrm{s})\end{array}$ & $\begin{array}{l}\text { Year } \\
(\mathrm{t})\end{array}$ & Event & $\begin{array}{l}\text { Post } \\
\text { Event }\end{array}$ & $\begin{array}{l}\text { Time to } \\
\text { Event }\end{array}$ & $\begin{array}{c}\mathrm{Lag} \\
4\end{array}$ & $\begin{array}{c}\mathrm{Lag} \\
3\end{array}$ & $\ldots$ & $\begin{array}{c}\text { Lead } \\
0\end{array}$ & $\begin{array}{c}\text { Lead } \\
1\end{array}$ & $\ldots$ & $\begin{array}{c}\text { Lead } \\
4\end{array}$ \\
\hline State A & 2000 & 2004 & 0 & -4 & 1 & 0 & $\ldots$ & 0 & 0 & $\cdots$ & 0 \\
\hline State A & 2001 & 2004 & 0 & -3 & 0 & 1 & $\ldots$ & 0 & 0 & $\ldots$ & 0 \\
\hline State A & 2002 & 2004 & 0 & -2 & 0 & 0 & $\ldots$ & 0 & 0 & $\ldots$ & 0 \\
\hline State A & 2003 & 2004 & 0 & -1 & 0 & 0 & $\ldots$ & 0 & 0 & $\ldots$ & 0 \\
\hline State A & 2004 & 2004 & 1 & 0 & 0 & 0 & $\ldots$ & 1 & 0 & $\ldots$ & 0 \\
\hline State A & 2005 & 2004 & 1 & 1 & 0 & 0 & $\ldots$ & 0 & 1 & $\ldots$ & 0 \\
\hline State A & 2006 & 2004 & 1 & 2 & 0 & 0 & $\ldots$ & 0 & 0 & $\ldots$ & 0 \\
\hline State A & 2007 & 2004 & 1 & 3 & 0 & 0 & $\ldots$ & 0 & 0 & $\ldots$ & 0 \\
\hline State A & 2008 & 2004 & 1 & 4 & 0 & 0 & $\ldots$ & 0 & 0 & $\ldots$ & 1 \\
\hline State A & 2009 & 2004 & 1 & 5 & 0 & 0 & $\cdots$ & 0 & 0 & $\ldots$ & 1 \\
\hline State B & 2000 & 2005 & 0 & -5 & 1 & 0 & $\cdots$ & 0 & 0 & $\cdots$ & 0 \\
\hline State B & 2001 & 2005 & 0 & -4 & 1 & 0 & $\ldots$ & 0 & 0 & $\ldots$ & 0 \\
\hline State B & 2002 & 2005 & 0 & -3 & 0 & 1 & $\ldots$ & 0 & 0 & $\ldots$ & 0 \\
\hline State B & 2003 & 2005 & 0 & -2 & 0 & 0 & $\ldots$ & 0 & 0 & $\ldots$ & 0 \\
\hline State B & 2004 & 2005 & 0 & -1 & 0 & 0 & $\ldots$ & 0 & 0 & $\ldots$ & 0 \\
\hline State B & 2005 & 2005 & 1 & 0 & 0 & 0 & $\ldots$ & 1 & 0 & $\ldots$ & 0 \\
\hline State B & 2006 & 2005 & 1 & 1 & 0 & 0 & $\ldots$ & 0 & 1 & $\ldots$ & 0 \\
\hline State B & 2007 & 2005 & 1 & 2 & 0 & 0 & $\ldots$ & 0 & 0 & $\ldots$ & 0 \\
\hline State B & 2008 & 2005 & 1 & 3 & 0 & 0 & $\ldots$ & 0 & 0 & $\ldots$ & 0 \\
\hline State B & 2009 & 2005 & 1 & 4 & 0 & 0 & $\ldots$ & 0 & 0 & $\ldots$ & 1 \\
\hline State C & 2000 & . & 0 & . & 0 & 0 & $\cdots$ & 0 & 0 & $\cdots$ & 0 \\
\hline State C & 2001 & . & 0 & . & 0 & 0 & $\ldots$ & 0 & 0 & $\ldots$ & 0 \\
\hline State C & 2002 & . & 0 & . & 0 & 0 & $\ldots$ & 0 & 0 & $\ldots$ & 0 \\
\hline State C & 2003 & . & 0 & . & 0 & 0 & $\cdots$ & 0 & 0 & $\cdots$ & 0 \\
\hline State C & 2004 & . & 0 & 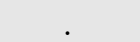 & 0 & 0 & $\ldots$ & 0 & 0 & $\ldots$ & 0 \\
\hline State C & 2005 & . & 0 & 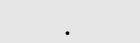 & 0 & 0 & $\ldots$ & 0 & 0 & $\ldots$ & 0 \\
\hline State C & 2006 & . & 0 & 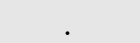 & 0 & 0 & $\ldots$ & 0 & 0 & $\ldots$ & 0 \\
\hline State C & 2007 & . & 0 & . & 0 & 0 & $\ldots$ & 0 & 0 & $\ldots$ & 0 \\
\hline State C & 2008 & . & 0 & . & 0 & 0 & $\ldots$ & 0 & 0 & $\ldots$ & 0 \\
\hline State C & 2009 & & 0 & . & 0 & 0 & $\ldots$ & 0 & 0 & $\ldots$ & 0 \\
\hline State D & 2000 & 2007 & 0 & -7 & 1 & 0 & $\cdots$ & 0 & 0 & $\cdots$ & 0 \\
\hline State D & 2001 & 2007 & 0 & -6 & 1 & 0 & $\ldots$ & 0 & 0 & $\ldots$ & 0 \\
\hline State D & 2002 & 2007 & 0 & -5 & 1 & 0 & $\ldots$ & 0 & 0 & $\ldots$ & 0 \\
\hline State D & 2003 & 2007 & 0 & -4 & 1 & 0 & $\ldots$ & 0 & 0 & $\ldots$ & 0 \\
\hline State D & 2004 & 2007 & 0 & -3 & 0 & 1 & $\ldots$ & 0 & 0 & $\ldots$ & 0 \\
\hline State D & 2005 & 2007 & 0 & -2 & 0 & 0 & $\ldots$ & 0 & 0 & $\ldots$ & 0 \\
\hline State D & 2006 & 2007 & 0 & -1 & 0 & 0 & $\ldots$ & 0 & 0 & $\ldots$ & 0 \\
\hline State D & 2007 & 2007 & 1 & 0 & 0 & 0 & $\ldots$ & 1 & 0 & $\ldots$ & 0 \\
\hline State D & 2008 & 2007 & 1 & 1 & 0 & 0 & $\ldots$ & 0 & 1 & $\ldots$ & 0 \\
\hline State D & 2009 & 2007 & 1 & 2 & 0 & 0 & $\ldots$ & 0 & 0 & $\ldots$ & 0 \\
\hline
\end{tabular}

Table 1: A Stylized Example 
A stylized example of such a setting is provided in Table 1. We consider four states

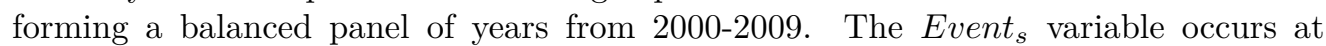
different times in different states, and in the case of one state, does not occur. Here both four lags and four leads are included, such that $J=K=4$. Lag and Lead 4 (exclusively) are switched on for periods in which the "Time to Event" exceeds 4 lags or leads respectively.

States in which the event never occurs (such as State C in Table 1) act as pure controls. These units have 0s in all lag and lead terms, and act as the counterfactual on which the estimation of impacts is based. Differences between these pure controls states and states which adopt the event of interest are anchored at 0 in the omitted base period, ie the first lag in equation 1 . Hence, lags and leads capture the difference between treated and control states, compared to the prevailing difference in the omitted base period. Unbiased estimation of post-event treatment effects thus relies fundamentally on the so called "parallel trends assumption". In the absence of treatment, it is assumed that treated and control states would have maintained similar differences as in the baseline period. For this reason, these models have been demonstrated to be under-identified, or identified only up to a linear trend, when all units adopt treatment at some point in time (Schmidheiny and Siegloch 2019; Borusyak and Jaravel 2018). Schmidheiny and Siegloch (2019) show that in this case, it is necessary to bin lags and leads beyond certain maximum lag $(J)$ and lead $(K)$ periods.

The panel event study is an extension of the standard two-way fixed effect (sometimes called difference-in-differences) model, where a single "Post Event" indicator is included for all periods posterior to the occurrence of the event in treated states. This is simply:

$$
y_{s t}=\alpha+\beta \text { PostEvent }_{s t}+\mu_{s}+\lambda_{t}+X_{s t}^{\prime} \Gamma+\varepsilon_{s t},
$$

where following the notation from (2)-(5), PostEvent ${ }_{s t}=\mathbb{1}\left[t \geq\right.$ Event $\left._{s}\right]$. Estimation of event specification 1 provides two key pieces of information not observable in this single-coefficient model. Firstly, the full set of event leads allows for the inspection of parallel trends in the pre-treatment period. While this does not provide evidence that the units in which the event was adopted and not adopted would have necessarily followed similar trends in the post-reform period (Kahn-Lang and Lang 2019) (which is the identifying assumption of these models), if trends in treated and untreated areas were not parallel even pre-event, it is unlikely that they would be parallel post-event. Secondly, the policy lags allow for inspection of the temporal nature of treatment effects, noting for example, any dynamics in the appearance of effects, for example growing or shrinking over time, and whether effects are transitory or permanent.

A developing literature including papers by de Chaisemartin and D'Haultfoeuille (2019); Callaway and Sant'Anna (2018); Goodman-Bacon (2018) point to challenges in interpreting the estimated $\widehat{\beta}$ from two-way fixed effects models when treatment effects are heterogeneous (across either groups or time periods). Goodman-Bacon (2018) for example, demonstrates that treatment effects which are heterogeneous in time since treatment in contexts where treatments are adopted in different time periods in different groups, can result in estimates which are biased away from a weighed average of the 
average treatment effect on the treated, a problem which is resolved in the panel event study design. However, results from Abraham and Sun (2018) suggest that specific types of heterogeneity concerns remain even in panel event study models examined here. In particular, they note undesired weighting of treatment effects if there is heterogeneity across treatment groups in particular lag and lead terms. Other concerns exist in event study designs, such as possible inferential problems related to selective survival of models based on pre-trend tests (Roth 2019). The eventdd command will not account for corrections raised in these particular settings, as these are inherent to empirical estimation of panel event study designs. We do however note that there are a number of alternative estimators which are complementary to panel event study designs, and which should be considered as part of a complete estimation and testing procedure, such as the stacked DD procedure of Abraham and Sun (2018), sensitivity tests described in Roth (2019); Rambachan and Roth (2019), and alternative models to account for dynamic paths of treatment effects, such as those described in de Chaisemartin and D'Haultfoeuille (2019); Callaway and Sant'Anna (2018). As many of these have existing estimation libraries in some languages, when discussing the command syntax of eventdd in section 3 and examples of use in section 4 we discuss ways which eventdd and its returned objects have been designed to facilitate interaction with these other commands.

\subsection{Inference}

A standard inference concern where policies are assigned by some unit such as state, and outcomes are followed over time within these units, is related to potential serialcorrelation in the outcome variable over time (Bertrand et al. 2004). While the derivations from (Bertrand et al. 2004) are based on single-coefficient models of the form of equation 6 , the crux of the concern relates to high serial correlation in the outcome variable of interest, and relatively little change in the independent variables of interest. This setting is replicated in event study models described in equations 1-5. It is thus fundamental to account for this within-cluster correlation when conducting inference in such models.

The standard solution is to allow for within-cluster auto-correlation by using a cluster-robust variance-covariance estimator (CRVE) to estimate standard errors and confidence intervals on regression parameters. Such an estimator is provided as standard in Stata by specifying the vce (cluster clustvar) option in e(class) models. ${ }^{5}$ However, as has been extensively documented, standard CRVEs are only asymptotically valid, where the asymptotic behavior depends on the number of clusters $S \rightarrow \infty$ (see for

\footnotetext{
${ }^{5}$ Denoting the matrix of $k$ independent variables as $\boldsymbol{X}$, the dependent variable vector as $\boldsymbol{y}$ and the vector of regression coefficients as $\boldsymbol{\beta}$, this CRVE is estimated in Stata for a linear regression model with $N$ observations in $S$ clusters as:

$$
\widehat{V}_{C R}(\widehat{\boldsymbol{\beta}})=\left(\boldsymbol{X}^{\prime} \boldsymbol{X}\right)^{-1}\left(\sum_{s=1}^{S} \boldsymbol{X}_{s} \tilde{\boldsymbol{u}}_{s} \tilde{\boldsymbol{u}}_{s}^{\prime} \boldsymbol{X}_{s}^{\prime}\right)\left(\boldsymbol{X}^{\prime} \boldsymbol{X}\right)^{-1} .
$$

Here $\tilde{\boldsymbol{u}}_{s}=\sqrt{c} \hat{\boldsymbol{u}}_{s}$, where $c$ is a small sample correction $c=(S /(S-1)) \times((N-1) /(N-k))$ and $\hat{\boldsymbol{u}}=\boldsymbol{y}-\boldsymbol{X} \widehat{\boldsymbol{\beta}}$ are standard regression residuals (Cameron et al. 2008).
} 
example the comprehensive review in Cameron and Miller (2015)). When standard clustering is used based on 'too few' clusters, the CRVE is generally downward-biased, resulting in over-rejection of null hypotheses. This bias can be severe (Cameron and Miller 2015; Mackinnon and Webb 2018).

In practice, knowing how many clusters it 'too few' depends on a number of factors. While there are rules of thumb such as the rule of 42 laid out in Angrist and Pischke (2008) which suggests that standard clustering provides a good approximation if $S \geq 42$ clusters, the performance of these methods under simulation has been shown to depend also on the relative size of clusters (Mackinnon and Webb 2017). A range of results surveyed in Cameron and Miller (2015) leads to their suggestion that if one is analyzing data with fewer than 50 clusters in a state-year panel (such as the case with panel-event studies), alternative inference methods should be considered.

In this case where the quasi-experimental set-up is based on fewer than around 50 clusters, the wild cluster bootstrap has been documented to be a successful resamplingbased method to take account of auto-correlation in variables underlying panel event studies, even in cases with fewer clusters (see eg Cameron et al. (2008); Cameron and Miller (2015); Roodman et al. (2019)). This has been efficiently implemented in Stata as described in Roodman et al. (2019), and programmed for Stata as boottest (Roodman 2015). Finally, note that in the case of very few clusters, and in particular few clusters where an event occurs, inference is further complicated. In cases such as this a number of potential solutions have been proposed, such as those described in Mackinnon and Webb (2018); Conley and Taber (2011). As we lay out in the following sections, the eventdd command allows simple access to various inference options depending on the context of interest, including standard clustering, bootstrap, and wild cluster bootstrap in various guises based on both Stata's native CRVE procedures, as well as the userwritten boottest command.

\section{The eventdd command}

Panel event studies can be implemented in Stata using the following command syntax:

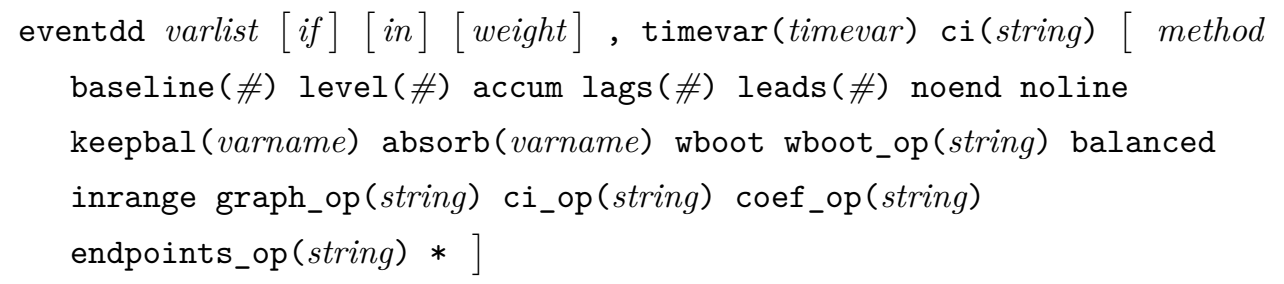

The required varlist should first specify the dependent variable of interest, and then all controls including fixed effects to be included in the panel event study model (equation 1), but not including lags and leads, that should be entered in the regression. The method option specifies the estimation procedure for the underlying model, and can be ols (ordinary least squares), fe (fixed effects) or hdfe (absorbing multiple levels of fixed 
effects with the user-written reghdfe command). If no estimation method is specified, ols is used by default. In the case of fixed effects ( $\mathrm{fe}$ ) or high-dimension fixed effect (hdfe) models, fixed effects can be absorbed (as discussed in the options below), and as such need not be entered in the standard varlist syntax. In the case of $f e$ specifications, data must first be xtset in Stata. Based on this syntax, eventdd takes care of the generation of all lag and lead terms, estimation and inference, and the production of an event study plot. Examples are provided in section 4 of this document.

\section{Options}

timevar is a required option. The time variable specified should contain a standardized value, where 0 corresponds to the time period in which the event of interest occurs for a given unit, -1 refers to one year prior to the event, 1 refers to one year following the event, and so forth. For any units in which the event does not occur (pure controls), this variable should contain missing values.

$\mathrm{ci}$ (string) is a required option indicating the type of graph the command will produce. The types of graphs available for the confidence intervals are rarea for an interval with area shading (see twoway_rarea), rcap for an interval with capped spikes (see twoway_rcap) and rline for an interval with lines (see twoway_rline). Only one type can be specified and all intervals will be the same type. The appearance can be modified with ci_op().

baseline(\#) Specifies the reference period for the event study, which is a baseline omitted category to which all other periods should be compared on the event study output. By default this value is set at -1 as in equation 1 .

level(\#) Specifies the confidence level, as a percentage, for confidence intervals. The default is level(95) or as set by set level. This sets the levels for confidence intervals in regression output, as well as the event study plot and matrices. This will also be passed to boottest if wild clustered standard errors are requested.

accum Specifies that all periods beyond some specified values should be accumulated into final points, indicated as $J$ and $K$ in equation 1. For example if accum is specified and lags(\#) and leads (\#) are both set equal to 10, a single coefficient will be displayed in regressions and graphical output capturing 10 or more periods prior/post reform. If accum is not specified, all possible lags and leads will be included in models and graphical output.

$\operatorname{lags}(\#)$ Indicates the maximum amount of pre-event periods to consider in the event study. This can only be specified if either accum, keepbal or inrange are also specified. Only integer values are permitted.

leads(\#) Indicates the maximum amount of post-event periods to consider in the event study. This can only be specified if either accum, keepbal or inrange are also specified. Only integer values are permitted.

noend Requests that accumulative end points are not shown on graphical output when 
the accum option is specified.

keepbal(varname) Specifies that only units which are balanced in the panel should be kept for estimation. Here varname indicates the panel variable (eg State) which indicates units. In this case "balance" refers to balance over calendar time. An alternative option (balanced), discussed below, allows for only balanced lags and leads relative to treatment to be considered in graphical output.

ols Requests that the event study model underlying graphical output should be estimated by OLS using Stata's regress command. In this case, unit-specific fixed effects and time-specific fixed effects must be included in the varlist indicated in the command syntax. This is the default estimation method.

fe Requests that the event study model underlying graphical output should be estimated by fixed-effects (within) estimation, using Stata's xtreg, fe command. In this case the data must be xtset prior to use, and unit-specific fixed effects should not be included in the varlist indicated in the command syntax. Time-specific fixed effects still need to be included in the varlist indicated in the command syntax.

hdfe Requests that the event study model underlying graphical output should be estimated using the user-written reghdfe command of Correia (2016) (if installed). If this option is specified, the absorb(varlist) option should also be specified to indicate which fixed effects should be controlled in the regression. Any fixed effects indicated in absorb(varlist) should not be included in the varlist indicated in the command syntax. This option cannot be used in combination with the wboot option.

absorb(varlist) This option is only required when specifying the hdfe estimation option. The varlist identifies fixed effects to be absorbed (such as unit fixed effects). Refer to reghdfe (if installed) for additional details.

wboot Indicates that inference in the event study plot produced by the command should be based on wild cluster bootstrapped standard errors. When indicated, confidence intervals for each lag and lead term will the be calculated using a wild cluster bootstrap. This requires the user-written boottest command of Roodman (2015) (if installed). This option may not be combined with the hdfe estimation option.

wboot_op(string) Allows for the inclusion of any other wild bootstrap option permitted in boottest, including seed(\#) to set the seed for simulation based calculations and replicate the confidence intervals, and bootclust (varname) to specify which variable(s) to cluster the wild bootstrap upon, among others. When setting the level (which is 95 by default), this should be indicated in the level option of the command, and this will be passed to wboot_op().

balanced Requests that only "balanced" lags and leads are plotted. This will produce a graph only showing lags and leads for which each treated unit has data, and as such, all coefficients plotted will be based on all units in the data. While only balanced lags and leads will be plotted, all units and time periods will be included in the estimation of the event study.

inrange Requests that only the specified lags and leads are plotted. While only lags and 
leads indicated in lags (\#) and leads (\#) will be plotted, all units and time periods will be included in the estimation of the event study.

noline Requests that the line before the event on the x-axis is not shown on graphical output.

graph_op(string) allows for the inclusion of any other graphing option permitted in twoway_options, including title_options, added_lines_options, axis_label_options, among others. This also allows for the use of alternative labels for graph axes. If not specified, a standard graphical output will be provided.

ci_op(string) Allows for the inclusion of any graphing option for the confidence intervals permitted in twoway_rarea, twoway_rcap or twoway_rline depending on the type of $\mathrm{CI}$ indicated in ci(); including area_options, line_options and connect_options, respectively. This does not allow the use of the general options of graph_op(). If not specified, a standard graphical output will be provided.

coef_op(string) Allows for the inclusion of any graphing option for the coefficients permitted in the scatter plot including marker_options and marker_label_options among others. This does not allow the use of the general options of graph_op(). If not specified, a standard graphical output will be provided.

endpoints_op(string) Allows for the inclusion of any graphing option for the end points coefficients permitted in scatter including marker_options, marker_label_options, among others. This is only available if specifying the accum and does not allow the use of the general options of graph_op(). If not specified, a standard graphical output will be provided.

* Any other estimation options permitted by regress, xtreg, or reghdfe can be included, and will be passed to the specified estimation command. This allows for the inclusion of clustered standard errors or other variance estimators such as bootstrap or jackknife estimators (see vce_option), as well as any other option relevant to the underlying estimation or inference in the panel event study regression model.

\section{Returned Objects}

eventdd stores the following in e():
Macros:
e (cmd)
eventdd
e(cmdline)
command as typed
e (depvar)
name of dependent variable
e (wtype)
weight type
e(clustvar)
e (vce)
name of cluster variable
e (vcetype)
vcetype specified in vce()
title used to label Std. Err.

Matrices: 
Damian Clarke and Kathya Tapia Schythe

$\begin{array}{ll}e(b) & \text { coefficient vector } \\ e(V) & \text { variance-covariance matrix of the estimators } \\ \text { e(leads) } & \text { all event leads, their lower bound, the point estimate, and } \\ & \text { their upper bound } \\ \text { e(lags) } & \text { all event lags, their lower bound, the point estimate, and } \\ \text { e(V_lags_leads) } & \text { variance-covariance matrix of the lags and leads estimators }\end{array}$

Note that methods related to event study models such as that described by Rambachan and Roth (2019) rely on access to point estimates and standard errors of lag and lead terms, which are available through the matrices returned here.

\section{Examples based on an Empirical Application}

We now provide a number of illustrations of the performance of eventdd to estimate the panel event study in empirical applications. We use data from Stevenson and Wolfers (2006) of the no-fault divorce reforms and female suicide in United States. These data have been used in other papers to demonstrate the functionality of recent advances in two-way fixed effect models (see Goodman-Bacon (2018)) and are drawn from examples used in documenting such methods when used in Stata (GoodmanBacon et al. 2019). ${ }^{6}$ The data consists of a balanced panel with 49 states observed from 1964 to 1996 with different timing of unilateral divorce reforms across the states.

The specification of the baseline two-way fixed effect "difference-in-differences" (DD)style model of female suicide on no-fault divorce reforms used is:

$$
\text { asmrs }_{s t}=\gamma_{s}+\lambda_{t}+\text { rpost }_{s t}+X_{s t}^{\prime} \Gamma+\varepsilon_{s t} .
$$

This is the analogue of equation 6 applied to this case in particular. Here, asmrs refers to the female suicide rate for all women in state $s$ at time $t, \gamma$ is a fixed effect by state, $\lambda$ is a temporal (year) fixed effect, post takes the value of 1 after the implementation of a no-fault divorce reform and $\varepsilon$ is a stochastic error. The controls $\left(X_{s t}\right)$ include Per-Capita Income (pcinc), Homicide Mortality (asmrh) and the Aid to Families with Dependent Children (AFDC) rate for a family of four (cases). Here $\tau$ is the parameter which captures the average impact of unilateral divorce on suicide rate assuming a standard DD parallel-trends assumption. ${ }^{7}$

\subsection{Estimation of the Panel Event Study}

In order to estimate a panel event study specification corresponding to the no fault divorce reform, the first step is to create the standardized version of the time to reform

\footnotetext{
${ }^{6}$ This data is available online at the following URL: http://www.damianclarke.net/stata/bacon_example.dta, and can be imported directly into Stata typing webuse set www.damianclarke.net/stata/ and then webuse bacon_example.dta.

${ }^{7}$ In reality, as laid out in section 2 , in the case that this effect is heterogeneous over time, this will not capture the ATE, but rather a weighted average of pair-wise comparisons (Goodman-Bacon 2018; de Chaisemartin and D'Haultfoeuille 2019; Callaway and Sant'Anna 2018).
} 
variable, presuming such a variable is not already available in the data. In this case in particular, the creation of the variable in Stata simply requires subtracting the reform period, called Event s $_{s}$ in section 2 (and _nfd, for 'no fault divorce', in the data) from the time period $t$, (called year in the data):

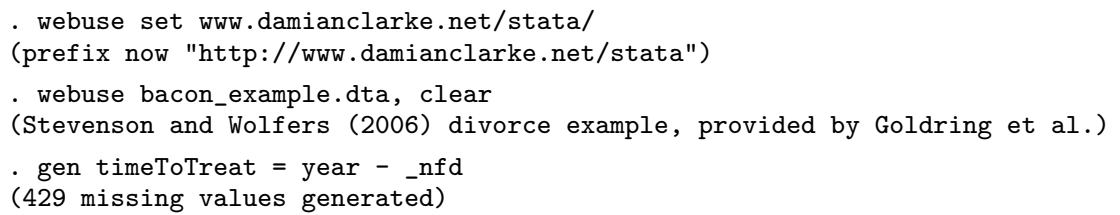

Note that as expected missing values are generated for states in which the reform is not adopted at any point in this period, and which act as pure controls in the panel event study.

The second step is to estimate the event study, as per equations 1-5. In this example, the general form of event study model including all lags and leads available is:

$$
\begin{aligned}
\text { asmrs }_{s t}=\alpha & +\beta_{21}(\operatorname{Lag} 21)_{s t}+\ldots+\beta_{2}(\operatorname{Lag} 2)_{s t} \\
& +\gamma_{0}(\operatorname{Lead} 0)_{s t}+\ldots+\gamma_{27}(\operatorname{Lead} 27)_{s t} \\
& +X_{s t}^{\prime} \Gamma+\mu_{s}+\lambda_{t}+\varepsilon_{s t}
\end{aligned}
$$

where as above asmrs is the female suicide rate for all women, and a series of $J=21$ Lags and $K=27$ Leads are considered relative to the event of interest (fully saturating the model). As is generally standard, the reference period is set as -1 : the period immediately preceding the adoption of the event in each state. Fixed effects for state and time are included as $\mu$ and $\lambda$, respectively.

The eventdd command provides a simple syntax to generate all necessary lags and leads for equation 8 , estimate the event study model, and plot point estimates and confidence intervals. The command requires two options: the standardized variable in timevar(timeToTreat) and also the type of confidence interval graph, for instance, we specify ci(rcap) for intervals indicated by capped error bars. Below we request that the command runs quietly (qui), however later in this section we document an example where full regression output is displayed:

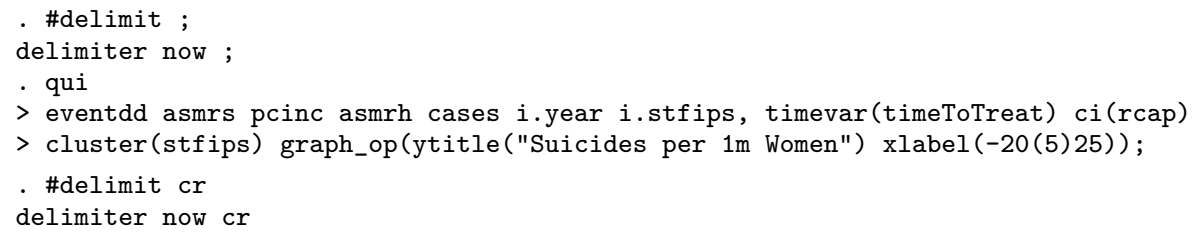

The command stores all event lags, their lower bound, the point estimate, and their upper bound. For example, if we wish to visualize the estimates on the full set of lags, as well as their upper and lower confidence intervals, we can simply examine the returned lags matrix: 


\begin{tabular}{|c|c|c|c|c|}
\hline & Lag & LB & Est & UB \\
\hline$r 21$ & 1 & 0 & 0 & 0 \\
\hline r20 & 2 & -5.6533713 & -.51573968 & 4.621892 \\
\hline $\mathrm{r} 19$ & 3 & -8.3800554 & -2.3125875 & 3.7548807 \\
\hline $\mathrm{r} 18$ & 4 & -4.6691194 & .22835743 & 5.1258345 \\
\hline $\mathrm{r} 17$ & 5 & -8.129283 & -2.7754233 & 2.5784359 \\
\hline $\mathrm{r} 16$ & 6 & -6.8603821 & -.75055814 & 5.3592658 \\
\hline$r 15$ & 7 & -10.123232 & -1.2564343 & 7.6103635 \\
\hline$r 14$ & 8 & -10.710829 & -2.7376504 & 5.2355275 \\
\hline$r 13$ & 9 & -12.330478 & -5.0007019 & 2.3290732 \\
\hline $\mathrm{r} 12$ & 10 & -11.226175 & -1.1506662 & 8.9248428 \\
\hline $\mathrm{r} 11$ & 11 & -17.51306 & -9.3819475 & -1.2508357 \\
\hline $\mathrm{r} 10$ & 12 & -14.165311 & -.04345011 & 14.078411 \\
\hline r9 & 13 & -10.856544 & -1.3885684 & 8.0794067 \\
\hline r8 & 14 & -6.3317404 & 4.3279953 & 14.987731 \\
\hline $\mathrm{r} 7$ & 15 & -7.7231503 & .84775668 & 9.418664 \\
\hline$r 6$ & 16 & -8.3638954 & -1.022577 & 6.3187418 \\
\hline r5 & 17 & -17.12311 & -4.4348736 & 8.2533636 \\
\hline$r 4$ & 18 & -10.074435 & -.51595128 & 9.0425329 \\
\hline r3 & 19 & -3.328351 & 8.8427277 & 21.013805 \\
\hline r2 & 20 & -34.54834 & -12.084179 & 10.379983 \\
\hline $\mathrm{r} 1$ & 21 & -31.114813 & -22.920727 & -14.72664 \\
\hline
\end{tabular}

Since we do not specify the estimation method, eventdd uses Stata's regress command to estimate the model by ordinary least-squares regression (if the ols option is specified, the same result is obtained). We can also request others estimators for the underlying event study model; if we specify the fe option, the model should be estimated with the fixed-effects (FE) estimator. ${ }^{8}$

In the same way, we can estimate the results efficiently absorbing multiple levels of fixed effects via the reghdfe command by specifying the hdfe option, which is quite useful when we have to control for many fixed effects. Note that in this case, the fixed effects of interest must be indicated using the absorb() option which is passed to the reghdfe command. For instance, if we wish to absorb the temporal and geographic fixed effects, the necessary syntax is as follows:

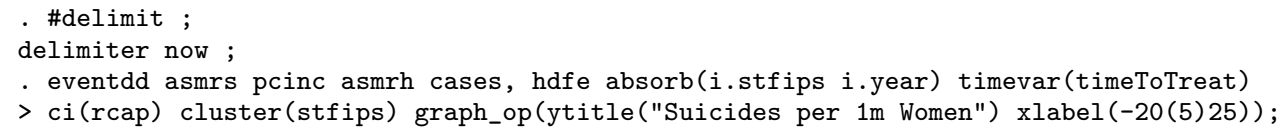

${ }^{8}$ Assuming the data has been xtset, this would simply be:

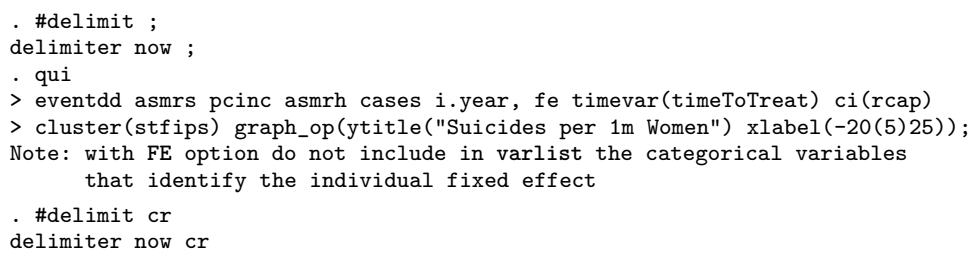

where note we no longer include the state fixed effects, as these will be taken care of given the panel fixed-effect (xtreg) estimator used to estimate the regression. 
Note: with HDFE option do not include in varlist the categorical variables that identify the fixed effects to be absorbed in absorb()

(MWFE estimator converged in 2 iterations)

warning: missing F statistic; dropped variables due to collinearity or too few clusters

HDFE Linear regression

Absorbing 2 HDFE groups

Statistics robust to heteroskedasticity

Number of clusters (stfips) =

$\begin{array}{llr}\text { Number of obs } & & 1,617 \\ \mathrm{~F}(51, \quad 48) & & . \\ \text { Prob }>\mathrm{F} & = & . \\ \text { R-squared } & = & 0.7212 \\ \text { Adj R-squared } & = & 0.6964 \\ \text { Within R-sq. } & = & 0.0731 \\ \text { Root MSE } & = & 10.8116\end{array}$

(Std. Err. adjusted for 49 clusters in stfips)

\begin{tabular}{|c|c|c|c|c|c|c|}
\hline asmrs & Coef. & $\begin{array}{l}\text { Robust } \\
\text { Std. Err. }\end{array}$ & $t$ & $P>|t|$ & {$[95 \%$ Conf. } & Interval] \\
\hline pcinc & -.0011046 & .0004071 & -2.71 & 0.009 & -.0019232 & -.0002861 \\
\hline asmrh & 1.08064 & .5968879 & 1.81 & 0.076 & -.119483 & 2.280764 \\
\hline cases & -190.3716 & 134.4991 & -1.42 & 0.163 & -460.8002 & 80.05698 \\
\hline $\operatorname{lag} 21$ & -22.92073 & 4.011063 & -5.71 & 0.000 & -30.98551 & -14.85594 \\
\hline lag20 & -12.08418 & 10.99637 & -1.10 & 0.277 & -34.19385 & 10.0255 \\
\hline $\operatorname{lag} 19$ & 8.842727 & 5.957829 & 1.48 & 0.144 & -3.13629 & 20.82174 \\
\hline $\operatorname{lag} 18$ & -.5159513 & 4.678946 & -0.11 & 0.913 & -9.923602 & 8.891699 \\
\hline $\operatorname{lag} 17$ & -4.434874 & 6.210982 & -0.71 & 0.479 & -16.92289 & 8.053142 \\
\hline $\operatorname{lag} 16$ & -1.022577 & 3.593627 & -0.28 & 0.777 & -8.248049 & 6.202895 \\
\hline $\operatorname{lag} 15$ & .8477567 & 4.19552 & 0.20 & 0.841 & -7.587901 & 9.283414 \\
\hline $\operatorname{lag} 14$ & 4.327995 & 5.218016 & 0.83 & 0.411 & -6.163529 & 14.81952 \\
\hline $\operatorname{lag} 13$ & -1.388568 & 4.634641 & -0.30 & 0.766 & -10.70714 & 7.930001 \\
\hline $\operatorname{lag} 12$ & -.0434501 & 6.912751 & -0.01 & 0.995 & -13.94247 & 13.85557 \\
\hline $\operatorname{lag} 11$ & -9.381948 & 3.980237 & -2.36 & 0.023 & -17.38475 & -1.379145 \\
\hline $\operatorname{lag} 10$ & -1.150666 & 4.932033 & -0.23 & 0.817 & -11.06718 & 8.765851 \\
\hline lag9 & -5.000702 & 3.587977 & -1.39 & 0.170 & -12.21481 & 2.213409 \\
\hline lag8 & -2.73765 & 3.902927 & -0.70 & 0.486 & -10.58501 & 5.10971 \\
\hline $\operatorname{lag} 7$ & -1.256434 & 4.34036 & -0.29 & 0.773 & -9.983313 & 7.470445 \\
\hline $\operatorname{lag} 6$ & -.7505582 & 2.990802 & -0.25 & 0.803 & -6.763968 & 5.262852 \\
\hline lag5 & -2.775423 & 2.620752 & -1.06 & 0.295 & -8.044798 & 2.493952 \\
\hline $\operatorname{lag} 4$ & .2283574 & 2.39735 & 0.10 & 0.925 & -4.591837 & 5.048552 \\
\hline $\operatorname{lag} 3$ & -2.312587 & 2.970068 & -0.78 & 0.440 & -8.28431 & 3.659136 \\
\hline lag2 & -.5157397 & 2.514907 & -0.21 & 0.838 & -5.572299 & 4.54082 \\
\hline lead0 & .2507466 & 2.722144 & 0.09 & 0.927 & -5.222491 & 5.723984 \\
\hline lead1 & -1.619351 & 2.941537 & -0.55 & 0.585 & -7.533709 & 4.295006 \\
\hline lead2 & -1.687107 & 3.898178 & -0.43 & 0.667 & -9.524919 & 6.150706 \\
\hline lead3 & -.7444709 & 2.862572 & -0.26 & 0.796 & -6.500058 & 5.011116 \\
\hline lead4 & -2.956354 & 2.832628 & -1.04 & 0.302 & -8.651735 & 2.739027 \\
\hline lead5 & -2.377841 & 2.75474 & -0.86 & 0.392 & -7.916617 & 3.160935 \\
\hline lead6 & -3.311888 & 3.568157 & -0.93 & 0.358 & -10.48615 & 3.862372 \\
\hline lead7 & -5.136502 & 3.401946 & -1.51 & 0.138 & -11.97657 & 1.70357 \\
\hline lead8 & -6.991146 & 3.086374 & -2.27 & 0.028 & -13.19672 & -.785575 \\
\hline lead9 & -4.82321 & 3.089481 & -1.56 & 0.125 & -11.03503 & 1.388607 \\
\hline lead10 & -8.814158 & 3.6746 & -2.40 & 0.020 & -16.20244 & -1.42588 \\
\hline lead11 & -7.27331 & 3.631759 & -2.00 & 0.051 & -14.57545 & .0288304 \\
\hline lead12 & -6.151559 & 4.089512 & -1.50 & 0.139 & -14.37407 & 2.070957 \\
\hline lead13 & -8.276837 & 3.946249 & -2.10 & 0.041 & -16.2113 & -.3423707 \\
\hline lead14 & -6.593221 & 3.867273 & -1.70 & 0.095 & -14.3689 & 1.182453 \\
\hline lead15 & -7.850839 & 4.070836 & -1.93 & 0.060 & -16.0358 & .3341258 \\
\hline lead 16 & -7.234422 & 4.270836 & -1.69 & 0.097 & -15.82151 & 1.35267 \\
\hline lead 17 & -8.516898 & 4.344278 & -1.96 & 0.056 & -17.25166 & .2178592 \\
\hline lead18 & -9.991582 & 3.758781 & -2.66 & 0.011 & -17.54912 & -2.434046 \\
\hline lead19 & -11.53613 & 3.861769 & -2.99 & 0.004 & -19.30074 & -3.771526 \\
\hline
\end{tabular}


Damian Clarke and Kathya Tapia Schythe

\begin{tabular}{r|rrrrrr} 
lead20 & -9.219165 & 4.501869 & -2.05 & 0.046 & -18.27078 & -.167551 \\
lead21 & -10.79088 & 4.417864 & -2.44 & 0.018 & -19.67359 & -1.908173 \\
lead22 & -10.65478 & 4.608349 & -2.31 & 0.025 & -19.92049 & -1.389076 \\
lead23 & -12.08658 & 5.29214 & -2.28 & 0.027 & -22.72714 & -1.446016 \\
lead24 & -10.67796 & 6.147523 & -1.74 & 0.089 & -23.03838 & 1.682466 \\
lead25 & -10.26777 & 7.459044 & -1.38 & 0.175 & -25.26518 & 4.729644 \\
lead26 & -16.69255 & 10.54234 & -1.58 & 0.120 & -37.88934 & 4.504239 \\
lead27 & -.4344752 & 8.147106 & -0.05 & 0.958 & -16.81533 & 15.94638 \\
_cons & 85.59069 & 10.90519 & 7.85 & 0.000 & 63.66433 & 107.5171 \\
\hline
\end{tabular}

Absorbed degrees of freedom:

\begin{tabular}{|c|c|c|c|c|c|}
\hline Absorbed FE & Categories & - Redundant & $=\mathrm{Num}$ & & oefs \\
\hline stfips & 49 & 49 & & 0 & $*$ \\
\hline year & 33 & 0 & & 33 & \\
\hline
\end{tabular}

* = FE nested within cluster; treated as redundant for DoF computation

. \#delimit cr

delimiter now cr

The standard command output consists of the regression output, and the event study lag and lead coefficients along with their confidence intervals are plotted as in Figure 1. As discussed in Stevenson and Wolfers (2006), the event study plot provides evidence of a reduction in rates of female suicide following the passage of no fault divorce laws, with significant declines observed 8 years following reform passage. We note that in this specification where all possible lags and leads are included (the default behavior of eventdd), we do observe a number of significant differences in the pre-reform period, in lag 11, and lag 21. It is important to note however that these lags are sufficiently far from the time-period of treatment that not all treated states are observed, and so these significant declines likely owe to compositional changes in these variables. We discuss this further below, and limit analysis to balanced periods when discussing the balanced option of the command. Nevertheless, if desired, we can also formally test the joint significance of all the lag terms simultaneously with the hypothesis:

$$
H_{0}: \beta_{21}=\beta_{20}=\cdots=\beta_{2}=0 \quad \text { versus } \quad H_{1}: H_{0} \text { does not hold. }
$$

This can be simply assessed post-estimation using Stata's test command:

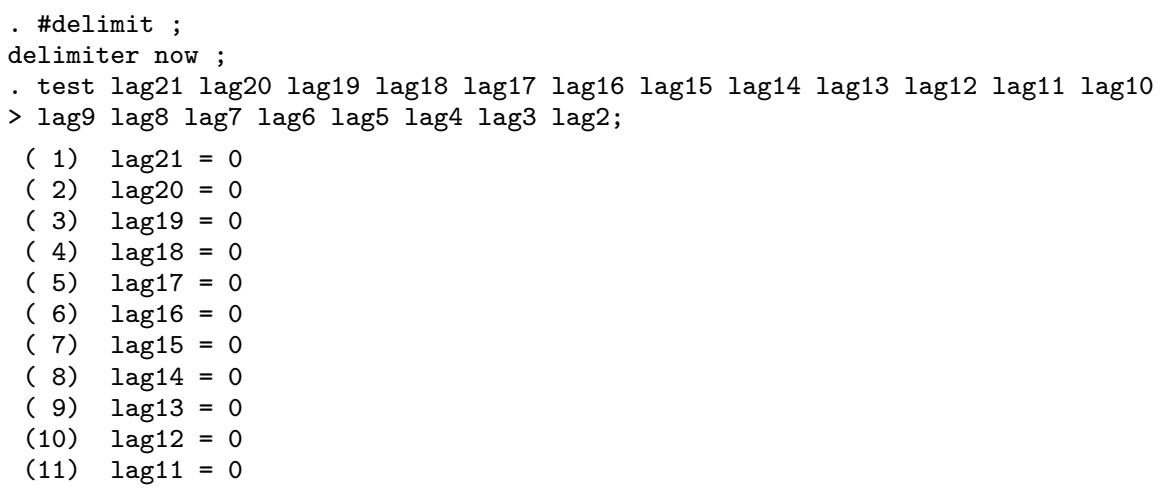




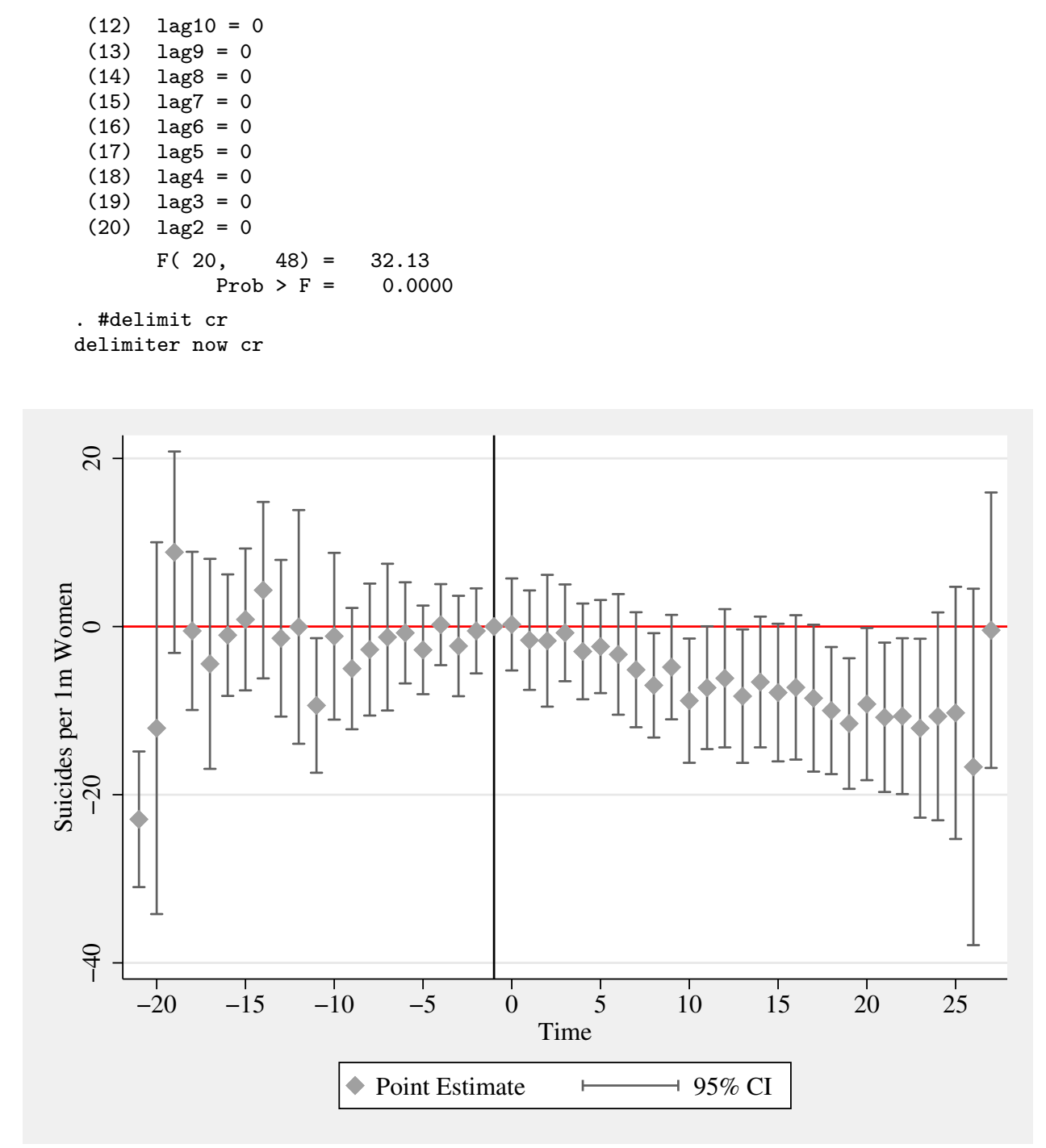

Figure 1: Event Study Example Based on No-fault Divorce Reforms

Notes: Event study model follows the No-Fault Divorce analysis described in (Stevenson and Wolfers 2006), and replication/extension of Goodman-Bacon (2018). Point estimates are displayed along with their $95 \%$ confidence intervals as described in equation 8 . The baseline (omitted) base period is 1 year prior to the adoption of the reform in each reforming state, indicated by the solid vertical line in the plot.

This 'fully-saturated' model where all possible lags and leads are plotted is the default output in the eventdd command. However many alternative estimation procedures are permitted, and indeed are likely preferred, for example to avoid the behavior observed 
above where lags and leads far from treatment will not be balanced given that only states adopting in certain early or late time periods will be observed in these lead/lag terms. Here we discuss a number of such alternatives, documenting their syntax in the eventdd command. Graphical output in each case is summarized in Figure 2.

Limiting Visualized Lags and Leads It may be a matter of interest to only show some lag/lead periods in the plot. For example, one such case discussed below relates to only plotting those lag/lead terms in which each treated state is observed. Generically, the inrange option allows for specifying that only certain coefficients and CIs should be included in plot. We note here that in this case, the underlying regression model will include all periods as in the first case, and as such, these lag/lead terms will simply correspond to a restricted range from Figure 1. For instance, if we want to show only the results between the time periods -10 and 10 , the command will be:

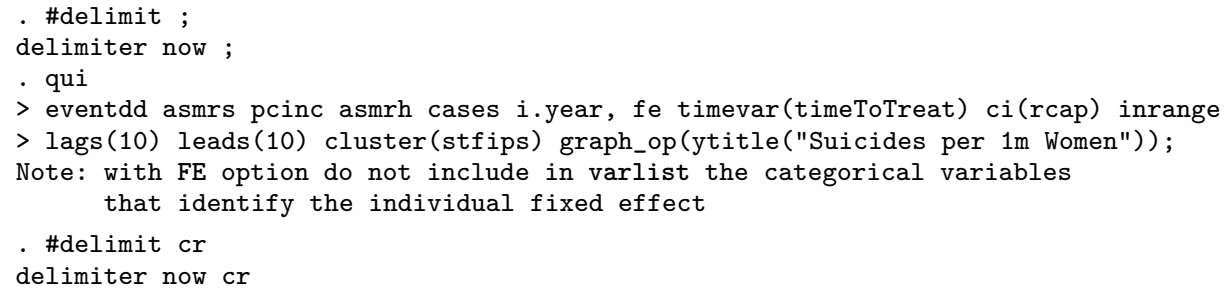

The output in this case is displayed in Figure 2(a). A special case of plotting limited lags/leads consists of the case in which one only wishes to show coefficients and CIs for which all states have a lag and lead term. We refer to this as a balanced plot, which can be produced quite simply using the balanced option. In this case, while all lags and leads are included in the underlying panel event study model, and only certain periods are plotted on the graph (like inrange), we do not need to know a priori which periods are balanced, as eventdd automatically identifies them. As panel (b) shows, in our case the balanced periods comprise periods between 5 years pre-reform and 11 years post-reform. ${ }^{9}$ In this case, the syntax simply requires indicating the balanced option:

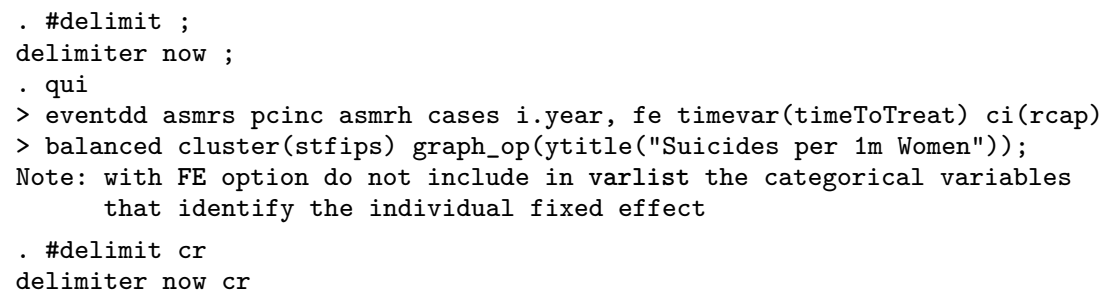

\footnotetext{
${ }^{9}$ This can also be seen quite simply by tabulating ( $t a b$ ) the time variable used to indicate time to treatment. For example, in the case of the timeToTreat variable indicating relative time to the arrival of the divorce reform, we observe that for each of lags -5 up until lead 11, all periods are observed in the 36 states in which the event occurs. However, earlier lags and later leads are only observed in a sub-sample of states given that for others, these lags (leads) are earlier (later) than the first (last) year in the data sample.
} 
Restricting Samples or Accumulating Lags/Leads In contrast to simply focusing on particular coefficients in the un-altered baseline model, one may wish to work with particular sub-samples which meet inclusion criteria, or accumulate lags and leads into periods which exceed some defined time, as an alternative way to avoid unbalanced lags and leads, as well as to avoid problems related to under-identification where all units are treated (Schmidheiny and Siegloch 2019). Consider the case where we wish to include 15 lags and 10 leads, but to only estimate the model with units that effectively have data for each of these periods. In the case of these data in particular which are yearly from 1964-1996, any units adopting no fault divorce reform between 1978 and 1996 will have (at least) 15 lags and 10 leads. Units adopting prior to 1978 will have fewer than 15 lags, and units adopting after 1996 will have fewer than 10 leads. To implement an estimation based on a balanced panel of observations with these lead/lag terms, the keepbal(varname) option is available, where varname indicates the panel unit over which balance should be applied (stfips in this case where the treatment unit is states). It is additionally necessary to explicitly indicate the period of interest for plotting within the balanced panel, for instance lags(15) and leads(10). This is all implemented in the below command.

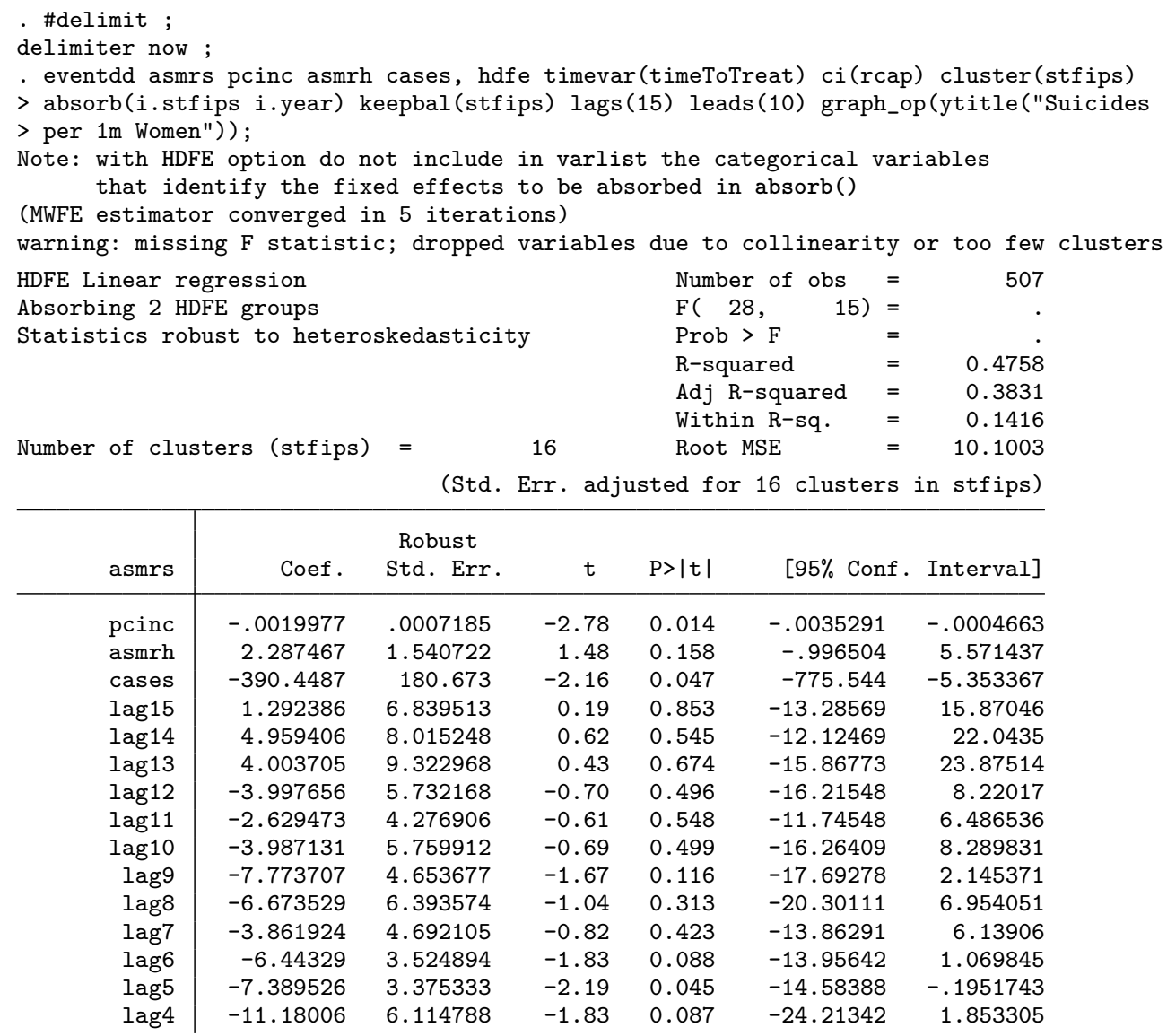


Damian Clarke and Kathya Tapia Schythe

\begin{tabular}{r|rrrrrr} 
lag3 & -2.146437 & 5.282028 & -0.41 & 0.690 & -13.40481 & 9.111939 \\
lag2 & -2.254288 & 3.599554 & -0.63 & 0.541 & -9.926557 & 5.41798 \\
lead0 & -3.458685 & 2.757283 & -1.25 & 0.229 & -9.335696 & 2.418325 \\
lead1 & -2.050697 & 3.988558 & -0.51 & 0.615 & -10.55211 & 6.450712 \\
lead2 & 3.8915 & 4.477937 & 0.87 & 0.399 & -5.652997 & 13.436 \\
lead3 & -5.949 & 5.387559 & -1.10 & 0.287 & -17.43231 & 5.53431 \\
lead4 & 1.151061 & 3.715023 & 0.31 & 0.761 & -6.767323 & 9.069445 \\
lead5 & 4.283462 & 6.478434 & 0.66 & 0.519 & -9.524993 & 18.09192 \\
lead6 & -3.08539 & 2.849672 & -1.08 & 0.296 & -9.159321 & 2.988541 \\
lead7 & 1.776614 & 7.27749 & 0.24 & 0.810 & -13.73499 & 17.28822 \\
lead8 & .7996726 & 5.648138 & 0.14 & 0.889 & -11.23905 & 12.83839 \\
lead9 & -1.215031 & 4.864573 & -0.25 & 0.806 & -11.58362 & 9.15356 \\
lead10 & -2.948988 & 4.776411 & -0.62 & 0.546 & -13.12967 & 7.231691 \\
- cons & 98.91732 & 14.70189 & 6.73 & 0.000 & 67.58098 & 130.2536 \\
\hline
\end{tabular}

Absorbed degrees of freedom:

\begin{tabular}{|r|rccc|}
\hline Absorbed FE & Categories & - Redundant & $=$ Num. Coefs \\
\hline stfips & 16 & 16 & 0
\end{tabular}$*$

* = FE nested within cluster; treated as redundant for DoF computation

. \#delimit cr

delimiter now cr

Given that we now restrict to only certain states based on their period of adoption (as well as un-adopting states) the lag and lead estimates will differ to those from the fully saturated model discussed previously. In the output of the above command, we observe that the estimation sample consists only of 507 observations for adopting states with balance in the indicated lags/leads, as well as states which do not adopt (versus 1617 observations in the full sample specification). The corresponding event study plot is presented in the panel (c), where we note that the considerable change in estimation sample (chosen simply for expositional reasons) produces quite different results.

An alternative way to work with the imbalance in standardized time periods is to stipulate that all periods beyond some specified values should be accumulated into final lag and lead points, as indicated in equation 2 and 5. This is implemented with the accum option. When this is specified, the panel event study is provided based on the number of leads and lags indicated in the lead(\#) and lag(\#) option respectively, accumulating all periods beyond these periods into the final lag and lead term. For instance, if we specify lags (-15) and leads (10), a single coefficient will capture the period -15 and earlier and the period 10 and later. This is illustrated in the following syntax, with the resulting graphical output presented in panel (d) of Figure 2.

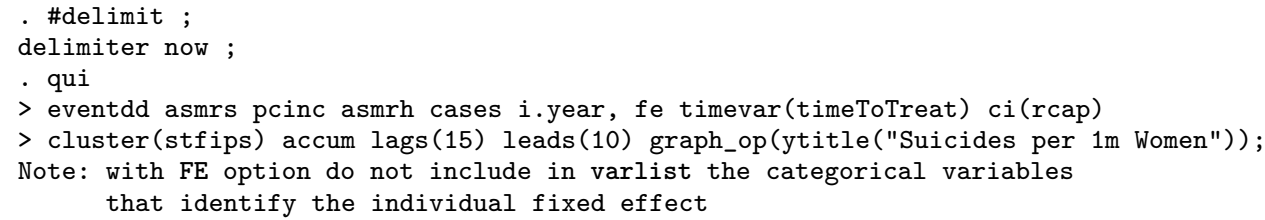


As these end points have a different interpretation to additional lags and leads, acting as an estimate of long-term impacts of the event for all periods beyond intermediate lags/leads, by default the end point estimates will be plotted in an alternative color. This behavior can be controlled fully using the endpoints_op() options, allowing for options such as marker styles and colors to be passed to the underlying scatter plot (additional discussion is provided in sub-section 4.3 of this paper). Alternatively, as documented below, the noend option can be invoked which will omit these final accumulative end points from graphical output, as shown in panel (e):

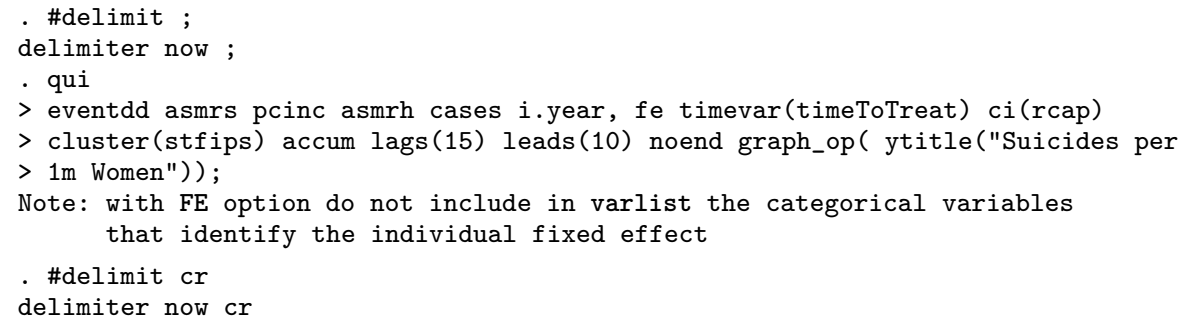

Finally, as discussed in section 2, the reference period for any panel event study estimated will be assumed to be the period immediately prior to the occurrence of the event in each state, unless otherwise indicated. This can be simply changed via the baseline(\#) option. While the choice of -1 as the baseline period is arbitrary, it is frequently adopted, and so alternative baseline periods should be based on some empirical or theoretical consideration, although both models will be equivalent up to a single constant shift. Below we provide the syntax setting an alternative baseline period, with all coefficients and standard errors referring to differences relative to 11 years prior to the event of interest. By default, the eventdd ado places a vertical reference line at period -1 to visually indicate the period immediately prior to the passage of the event. However, if this reference line is not desired, the noline option can be specified, as documented in Figure 2f. If one wishes to provide alternative reference periods, these can be passed directly to the graphing command. For example, to add an alternative reference line in period 0 one should specify graph_op(xline(0)).

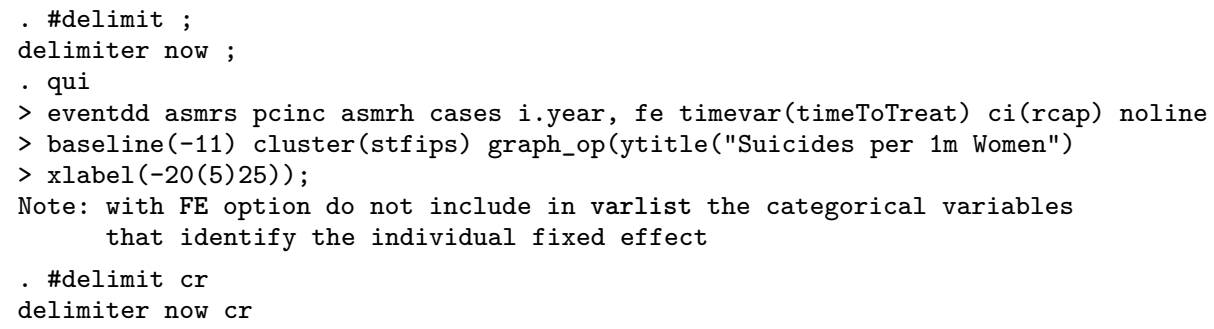



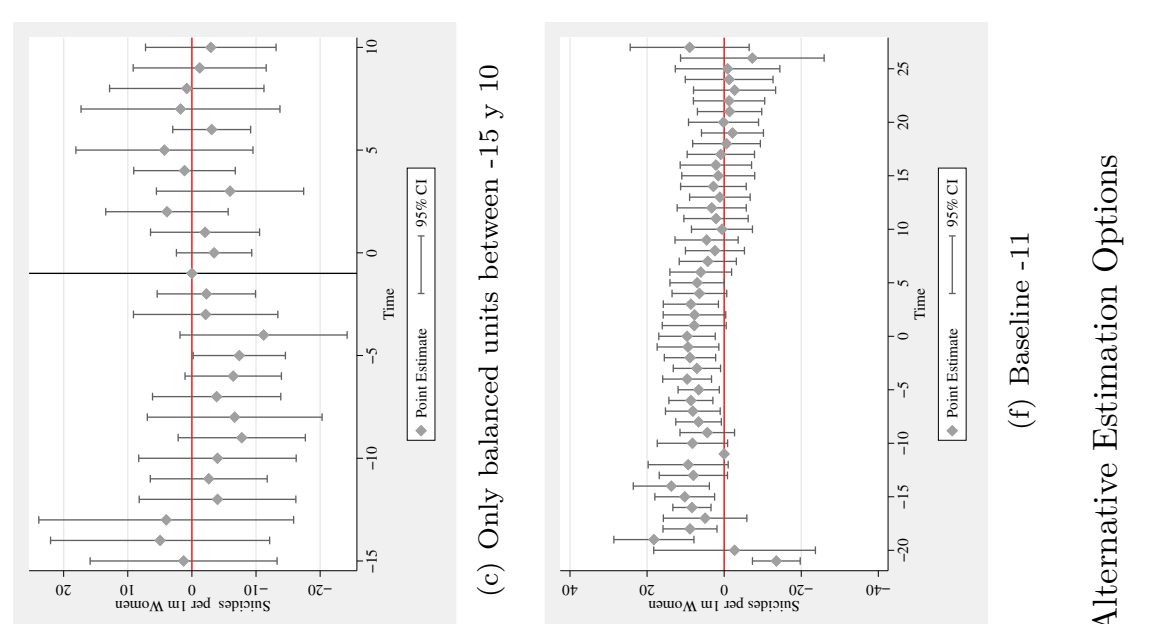

चี

尝

60 శี

:

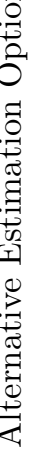

$\exists \bar{\pi} \stackrel{\square}{\square}$

.90

궁

:

च

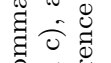

ठํ.

잉

政:
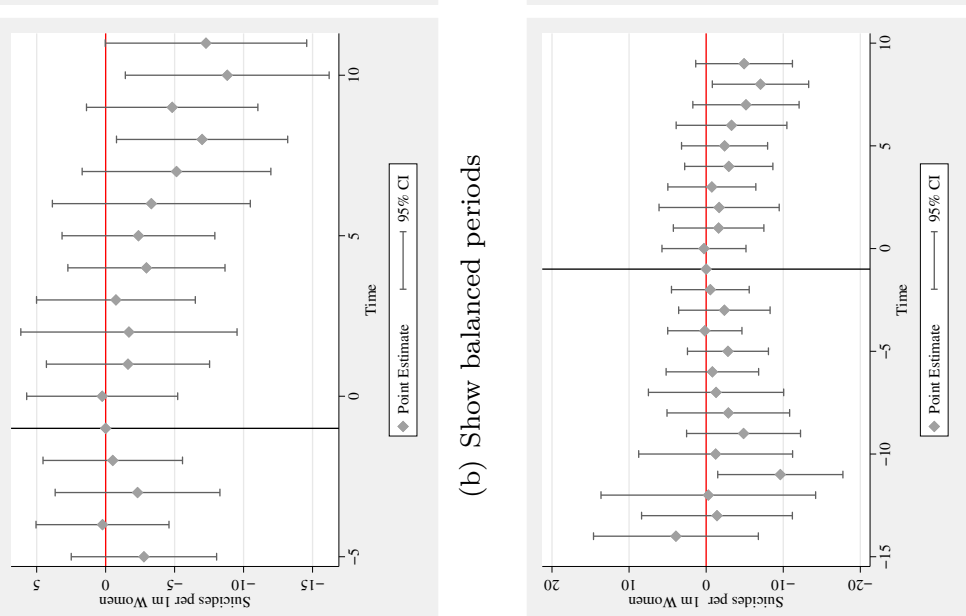

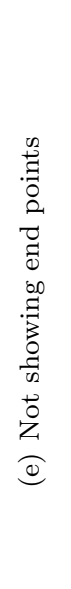

-1

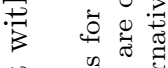

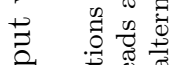

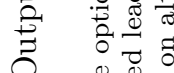

क

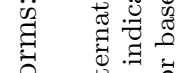

过

¿

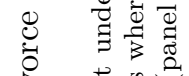

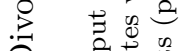

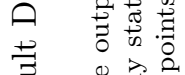

节 욜

氶
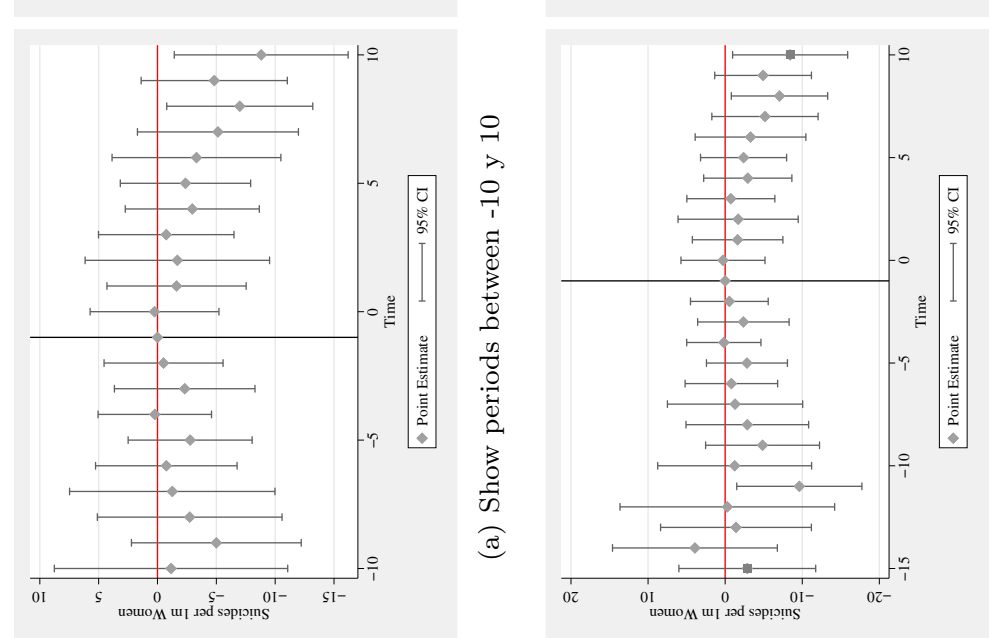

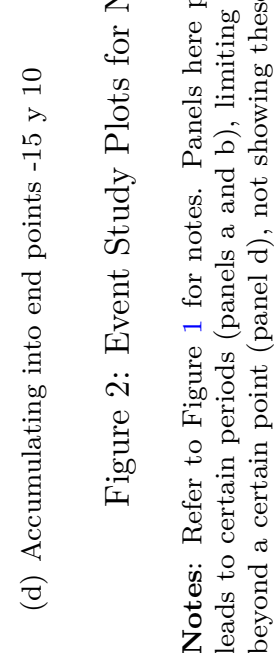




\subsection{Inference Options}

The previous sub-section describes a number of alternative estimation procedures which are potentially of relevance in the estimation of a panel event study design. However, as discussed in section 2 of this paper, there are a number of inference considerations which must be weighed when implementing a panel event study model. Up until this point, the command has always been implemented with cluster(stfips), indicating that a CRVE should be estimated, where clusters are based at the level of the state - the level at which the event is assigned in this case. As discussed in section 2.2, in this example based on 49 states, and hence 49 clusters, a CRVE is likely the appropriate inference mode for this model.

However, the eventdd command allows for inference using a wild clustered bootstrap as post-estimation procedure, via its interaction with the boottest command (provided this command is installed on the user's system). This is indicated by the wboot option, which by default assumes that a clustered wild bootstrap is desired, with the cluster variable indicated in the cluster() option. This is especially useful when there are few clusters in the panel. However, note that given that this procedures is based around bootstrap resampling, the inference procedure likely will take longer than inference based on Stata's native CRVE, and additionally, that the wboot option may not be combined with the hdfe estimation option. However, boottest offers considerable other benefits, including the option to undertake inference with two way clustering which may exhibit preferable size properties in the case of very few clusters (Mackinnon and Webb 2018). Any option which should be passed directly to boottest can be indicated in the wboot_op() option, as illustrated with the seed option below, ensuring replicability in pseudo-random bootstrap resamples if desired. Figure 3 contrasts the differences between the previous CRVE-based inference procedure with the wild cluster bootstrap inference procedure illustrated here.

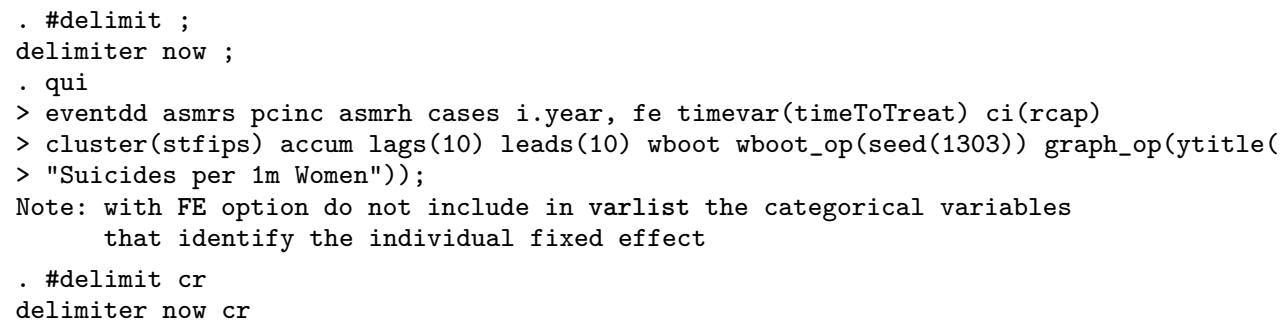

Finally, note that as standard, eventdd provides $95 \%$ confidence intervals in the command's output, returned objects, and the resulting graph and legend. The level() option allows for alternative levels to be indicated, where for example $90 \%$ CIs are requested below. Graphical output only differs in the confidence intervals provided (Figure 5(a) versus (b)).

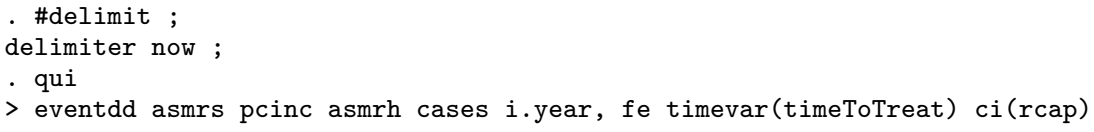




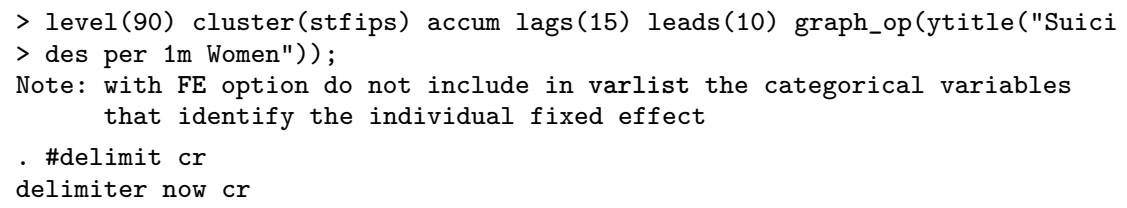

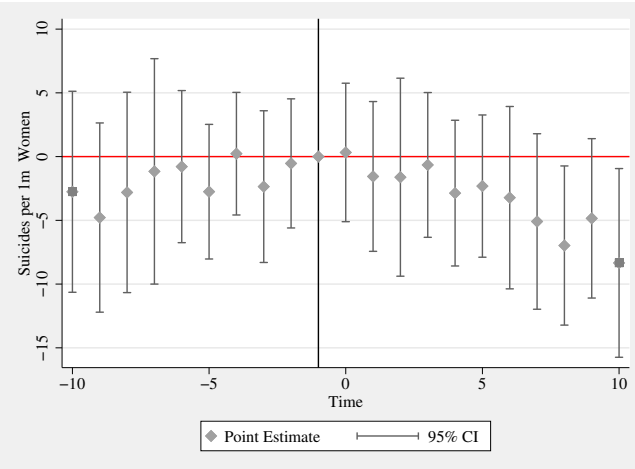

(a) Cluster-Robust VCE

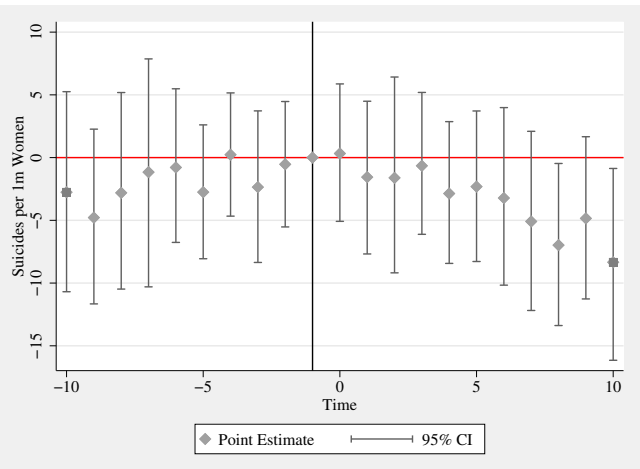

(b) Wild cluster boot-strapped, seed (1303)

Figure 3: Visualizing Alternative Inference Procedures for Event Study Models

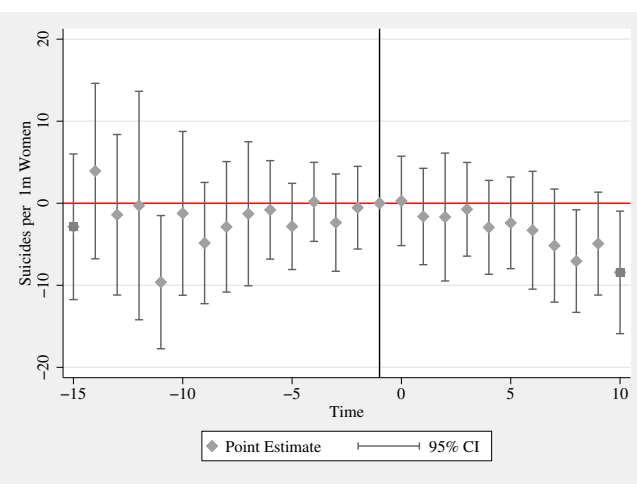

(a) $95 \%$ Confidence level

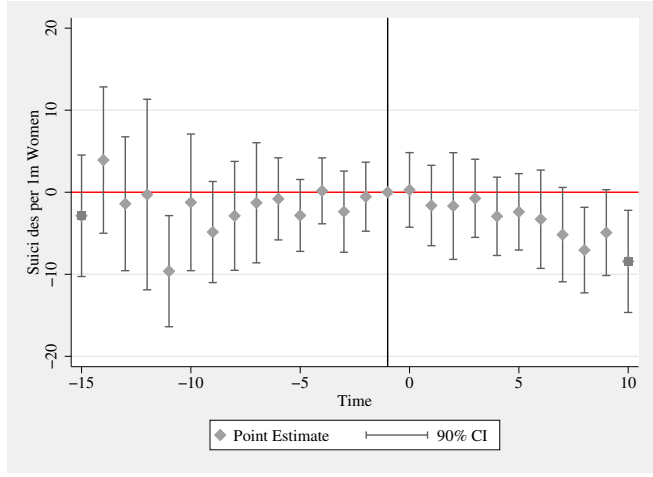

(b) $90 \%$ Confidence level

Figure 4: Default Event Study Plots with Alternative Confidence Intervals

\subsection{Altering Standard Appearance}

eventdd, allows for a number of ways to visualize the confidence intervals using a range of Stata's standard twoway graph types. The command requires that the user specify one of the following types of confidence intervals by specifying ci(rarea) for an 
interval with area shading, ci(rcap) for an interval with capped peaks, and ci(rline) for an interval with lines. Figure 5 shows the initial event study from Figure 1, however now with the three alternative types of plots available. One type must be specified, and this will be apply for all intervals displayed.

These graph types can be fully controlled using the ci_op() option, though the options included must be compatible with the actual type of confidence interval requested. The compatibility of options can be confirmed in Stata's help files for twoway rcap, twoway rarea, or twoway rline for each of the accepted ci() options. Similarly, we can specify any options desired for the graphing of the the coefficients in the plot with the coef_op() option and if we are accumulating periods into final points, we can specify graphing options for these points in endpoints_op(). In both cases, these accept any valid options for Stata's twoway scatter plot type. Finally, a graph_op() option allows for the inclusion of any general graphing options, such as alternative labelling schemes, graph schemes or title options. In Figure 6 we compare a standard output (left) with an alternative output (right) taking advantage of Stata's transparency options and alternative color schemes. The eventdd syntax used to generate Figure 7(b) is provided below, followed by the resulting output.

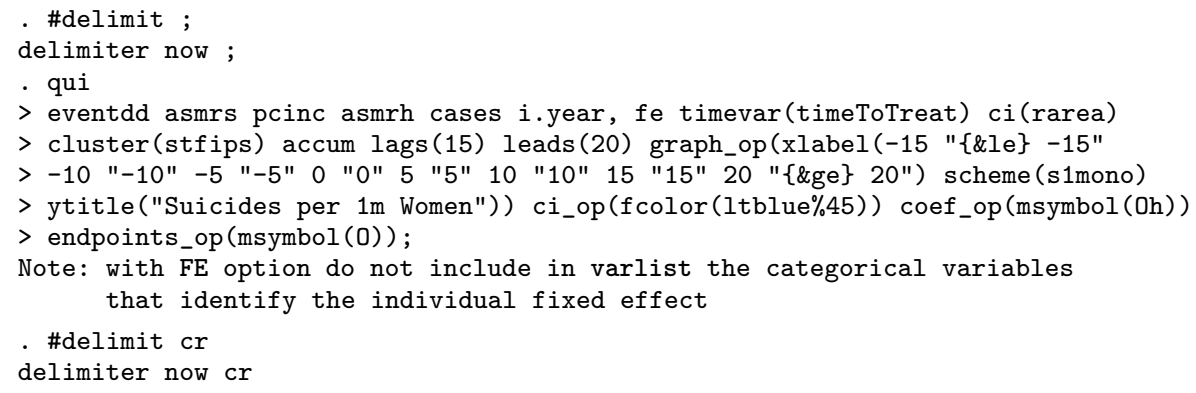

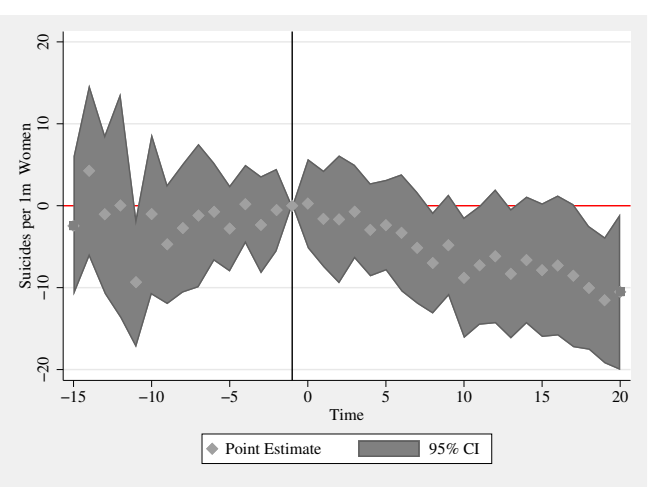

(a) Plot with standard appearance

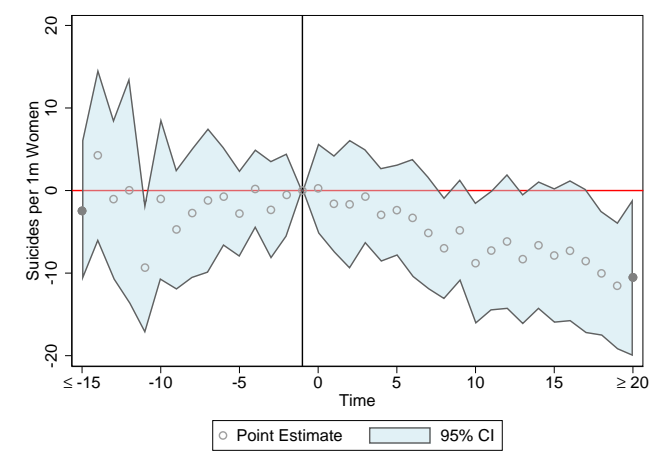

(b) Plot with alternative appearance options

Figure 6: Event study plots No-fault Divorce Reforms: Appearance options 


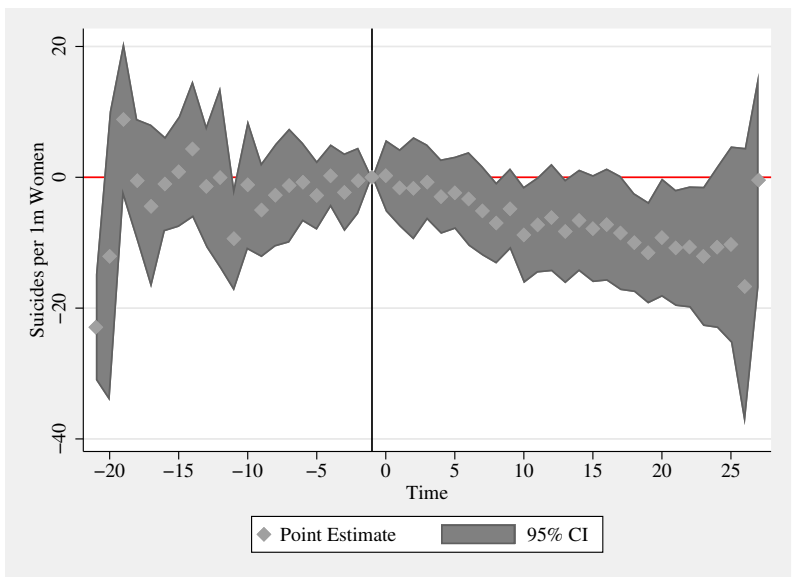

(a) ci (rarea): Interval with area shading

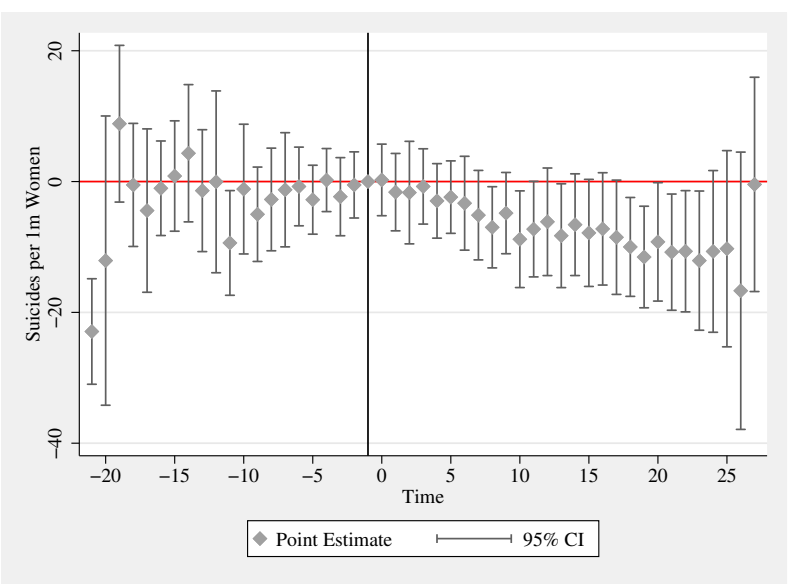

(b) ci (rcap): Interval with capped peaks

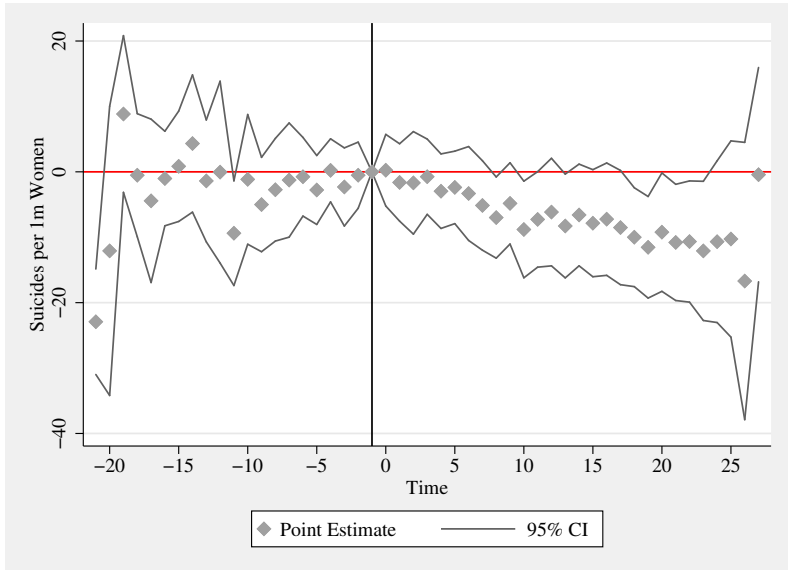

(c) ci(rline): Interval with lines

Figure 5: Alternative Visualization Options for Event Study Confidence Intervals 


\section{Conclusions}

The panel event study is an increasingly frequently used tool in the applied analysts' toolbox. It allows for the clear presentation of estimated impacts in quasi-experimental (observational) contexts, when one wishes to consider the impact of some event which occurs at (potentially) different times in different geographical areas. What's more while the discussion and examples provided in this paper are structured around geographical clustering of events (such as the application of divorce reforms studied in Stevenson and Wolfers (2006), and applied to demonstrate other two-way fixed effects methods (Goodman-Bacon 2018), this setting can similarly be applied where there is the temporal arrival of some event of interest in other dimensions, such as by age or other demographic groups.

In this paper we discuss a growing literature laying out panel event study designs, and introduce a flexible command eventdd which allows for their estimation and visualization in Stata. We introduce a number of estimation and inference concerns, and show how the command can simply deal with such concerns in an applied setting. While eventdd can be based on Stata's native routines such as regress or xtreg and cluster robust variance-covariance estimators, it is also able to interact with a number of extremely powerful user-written commands, allowing for extensions such as the efficient estimation of high-dimensional fixed effects equations, and the use of a wild cluster bootstrap for inference.

\section{References}

Abraham, S., and L. Sun. 2018. Estimating Dynamic Treatment Effects in Event Studies with Heterogeneous Treatment Effects.

Angrist, J. D., and J.-S. Pischke. 2008. Mostly Harmless Econometrics: An Empiricist's Companion. Princeton University Press.

Athey, S., and G. W. Imbens. 2018. Design-based Analysis in Difference-In-Differences Settings with Staggered Adoption. Working Paper 24963, National Bureau of Economic Research.

Bailey, M. J., O. Malkova, and Z. M. McLaren. 2018. Does Access to Family Planning Increase Children's Opportunities? Evidence from the War on Poverty and the Early Years of Title X. Journal of Human Resources . http://jhr.uwpress.org/content/early/2018/07/03/jhr.55.1.1216-8401R1.abstract.

Bertrand, M., E. Duflo, and S. Mullainathan. 2004. How Much Should We Trust Differences-In-Differences Estimates? The Quarterly Journal of Economics 119(1): 249-275.

Borusyak, K., and X. Jaravel. 2018. Revisiting Event Study Designs, with an Application to the Estimation of the Marginal Propensity to Consume. mimeo. https://scholar.harvard.edu/files/borusyak/files/event_studies_may8_website.pdf. 
Callaway, B., and P. H. C. Sant'Anna. 2018. Difference-in-Differences with Multiple Time Periods and an Application on the Minimum Wage and Employment. DETU Working Papers 1804, Department of Economics, Temple University. https://ideas.repec.org/p/tem/wpaper/1804.html.

Cameron, A., and D. Miller. 2015. A Practitioner's Guide to Cluster-Robust Inference. Journal of Human Resources 50(2): 317-372.

Cameron, A. C., J. B. Gelbach, and D. L. Miller. 2008. Bootstrap-based improvements for inference with clustered errors. Review of Economics and Statistics 90: 414-427.

de Chaisemartin, C., and X. D'Haultfoeuille. 2019. Two-way Fixed Effects Estimators with Heterogeneous Treatment Effects. NBER Working Papers 25904, National Bureau of Economic Research, Inc. https://ideas.repec.org/p/nbr/nberwo/25904.html.

Clarke, D., and K. Tapia Schythe. 2020. EVENTDD: Stata module to panel event study models and generate event study plots. https://EconPapers.repec.org/RePEc:boc:bocode:s458737.

Conley, T. G., and C. R. Taber. 2011. Inference with "Difference in Differences" with a Small Number of Policy Changes. The Review of Economics and Statistics 93(1): $113-125$.

Correia, S. 2016. Linear Models with High-Dimensional Fixed Effects: An Efficient and Feasible Estimator. Technical report. Working Paper. http://scorreia.com/research/hdfe.pdf.

Dimitrovová, K., J. Perelman, and M. Serrano-Alarcón. 2020. Effect of a national primary care reform on avoidable hospital admissions (2000-2015): A difference-in-difference analysis. Social Science \& Medicine 252: 112908. http://www.sciencedirect.com/science/article/pii/S0277953620301271.

Freyaldenhoven, S., C. Hansen, and J. M. Shapiro. 2019. Pre-event Trends in the Panel Event-Study Design. American Economic Review 109(9): 3307-38. http://www.aeaweb.org/articles?id=10.1257/aer.20180609.

Goodman-Bacon, A. 2018. Difference-in-Differences with Variation in Treatment Timing. Working Paper 25018, National Bureau of Economic Research. http://www.nber.org/papers/w25018.

Goodman-Bacon, A., T. Goldring, and A. Nichols. 2019. BACONDECOMP: Stata module to perform a Bacon decomposition of difference-in-differences estimation. Statistical Software Components, Boston College Department of Economics. https://ideas.repec.org/c/boc/bocode/s458676.html.

Kahn-Lang, A., and K. Lang. 2019. The Promise and Pitfalls of Differences-inDifferences: Reflections on 16 and Pregnant and Other Applications. Journal of Business \& Economic Statistics 0(0): 1-14. 
Mackinnon, J., and M. Webb. 2017. Wild Bootstrap Inference for Wildly Different Cluster Sizes. Journal of Applied Econometrics 32: 233-254.

_ 2018. The wild bootstrap for few (treated) clusters. The Econometrics Journal 21: $114-135$.

Pacicco, F., L. Vena, and A. Venegoni. 2018. Event Study Estimations Using Stata: The Estudy Command. The Stata Journal 18(2): 461-476.

Rambachan, A., and J. Roth. 2019. An Honest Approach to Parallel Trends.

Roodman, D. 2015. BOOTTEST: Stata module to provide fast execution of the wild bootstrap with null imposed. Statistical Software Components, Boston College Department of Economics. https://ideas.repec.org/c/boc/bocode/s458121.html.

Roodman, D., M. Ø. Nielsen, J. G. MacKinnon, and M. D. Webb. 2019. Fast and wild: Bootstrap inference in Stata using boottest. The Stata Journal 19(1): 4-60.

Roth, J. 2019. Pre-test with Caution: Event-study Estimates After Testing for Parallel Trends.

Schmidheiny, K., and S. Siegloch. 2019. On Event Study Designs and DistributedLag Models: Equivalence, Generalization and Practical Implications. IZA Discussion Papers 12079, Institute of Labor Economics (IZA).

Stevenson, B., and J. Wolfers. 2006. Bargaining in the Shadow of the Law: Divorce Laws and Family Distress*. The Quarterly Journal of Economics 121(1): 267-288.

Suhonen, T., and H. Karhunen. 2019. The intergenerational effects of parental higher education: Evidence from changes in university accessibility. Journal of Public Economics 176: $195-217$. http://www.sciencedirect.com/science/article/pii/S0047272719300945.

Venkataramani, A. S., E. F. Bair, R. L. O'Brien, and A. C. Tsai. 2020. Association Between Automotive Assembly Plant Closures and Opioid Overdose Mortality in the United States: A Difference-in-Differences Analysis. JAMA Internal Medicine 180(2): 254-262. https://doi.org/10.1001/jamainternmed.2019.5686.

\section{About the authors}

Damian Clarke is an Assistant Professor at The Department of Economics of The Universidad de Chile, a Research Fellow at IZA and an Associate at the Millennium Nucleus for the Study of the Life Course and Vulnerability.

Kathya Tapia Schythe is an Adjunct Professor in econometrics at The University of Santiago de Chile, and a young researcher associated with the Millennium Nucleus for the Study of the Life Course and Vulnerability.

\section{Acknowledgments}


Both authors acknowledge the financial support of the Universidad de Santiago de Chile, and the Millennium Nucleus for the Study of the Life Course and Vulnerability, funded by the Ministry of Economics of the Government of Chile. 